\title{
OPTIMAL MITIGATION POLICIES IN A PANDEMIC: SOCIAL DISTANCING AND WORKING FROM HOME
}

\author{
Callum J. Jones \\ Thomas Philippon \\ Venky Venkateswaran \\ Working Paper 26984 \\ http://www.nber.org/papers/w26984 \\ NATIONAL BUREAU OF ECONOMIC RESEARCH \\ 1050 Massachusetts Avenue \\ Cambridge, MA 02138 \\ April 2020
}

We are grateful for comments from seminar participants at the University of Chicago, NBER Summer Institute. The views expressed herein are those of the authors and should not be attributed to the IMF, its Executive Board, its management, or the National Bureau of Economic Research. First version: March 31, 2020.

NBER working papers are circulated for discussion and comment purposes. They have not been peer-reviewed or been subject to the review by the NBER Board of Directors that accompanies official NBER publications.

(C) 2020 by Callum J. Jones, Thomas Philippon, and Venky Venkateswaran. All rights reserved. Short sections of text, not to exceed two paragraphs, may be quoted without explicit permission provided that full credit, including $(\odot$ notice, is given to the source. 
Optimal Mitigation Policies in a Pandemic: Social Distancing and Working from Home

Callum J. Jones, Thomas Philippon, and Venky Venkateswaran

NBER Working Paper No. 26984

April 2020, Revised in October 2020

JEL No. E2,E6,I1

\begin{abstract}
$\underline{\text { ABSTRACT }}$
We study the response of an economy to an unexpected epidemic. Households mitigate the spread of the disease by reducing consumption, reducing hours worked, and working from home. Working from home is subject to learning-by-doing and the capacity of the health care system is limited. A social planner worries about two externalities, an infection externality and a healthcare congestion externality. Private agents' mitigation incentives are too weak and suffer from a fatalism bias with respect to future infection rates. The planner implements front-loaded mitigation policies and encourages working from home immediately. In our calibration, assuming a CFR of $1 \%$ and an initial infection rate of $0.1 \%$, private mitigation reduces the cumulative death rate from $2.5 \%$ of the initially susceptible population to about $1.75 \%$. The planner optimally imposes a drastic suppression policy and reduces the death rate to $0.15 \%$ at the cost of an initial drop in consumption of around $25 \%$.
\end{abstract}

Callum J. Jones

jonescallum@gmail.com

Thomas Philippon

New York University

Stern School of Business

44 West 4th Street, Suite 9-190

New York, NY 10012-1126

and NBER

tphilipp@stern.nyu.edu
Venky Venkateswaran

Stern School of Business

New York University

7-81 44 West 4th Street

New York, NY 10012

and Federal Reserve Bank of Minneapolis

and also NBER

vvenkate@stern.nyu.edu 


\section{Introduction}

The response to the Covid-19 crisis highlights the tension between health and economic outcomes. We propose a simple extension of the neoclassical model to quantify the tradeoffs and guide policy. When will the private sector engineer the right response, and when is there a need for policy intervention? We are particularly interested in understanding the nature and timing of policy responses. Which measures should be front-loaded and which ones should ramp up as the contagion progresses?

Our model has two building blocks: one for the dynamics of contagion, and one for consumption and production, including mitigation strategies (such as working from home). Our starting point is the classic SIR model of contagion used by public health specialists. Atkeson (2020b) provides a good summary of this framework. In a population of initial size $N$, the epidemiological state is given by the numbers of Susceptible $(S)$, Infected $(I)$, and Recovered $(R)$ people. By definition, the cumulative number of deaths is $D=N-S-I-R$. Infected people transmit the virus to susceptible people at a rate that depends on the nature of the virus and on the frequency of social interactions. Containment, testing, and social distancing reduce this later factor. The rates of recovery (transitions from $I$ to $R$ ), morbidity ( $I$ becoming severely or critically sick) and mortality (transition form $I$ to $D$ ) depend on the nature of the virus and on the quality of health care services. The quality of health services depends on the capacity of health care providers (ICU beds, ventilators) and the number of sick people. The economic side of the model focuses on three key decisions: consumption, labor supply, and working from home. We use a canonical representative-agent framework where members of a large household jointly make these decisions. Both consumption and work increase th risk of contagion, which is the key link between the economic and epidemiological blocks of the model. We endow agents with the option of working from home, which reduces contagion risk but comes at a cost in the form of productivity losses. These losses decline as agents accumulate experience in working from home.

We use a calibrated version of the model to study the reaction of private agents in equilibrium to the announcement of an outbreak as well as that of a social planner. Upon 
learning of the risks posed by the virus, households cut spending and labor supply and increase time spent working from home. Broadly, their mitigation efforts are proportionate to the risk of infection, which - all else equal - is proportional to the fraction of infected agents $I / N$. Private responses differ from those of the planner because of two externalities. The first is the usual infection externality: households only take into account the risk that they become infected, not the risk that they infect others. The other is is a congestion externality: agents do not internalize the fact that their actions can increase the fatality risk for others, through an overwhelmed healthcare system. Both these factors make private mitigation lower than the socially optimal level. We show that this wedge between private and social incentives can be particularly severe early on in the outbreak. When private agents become aware of the disease, the possibility of future infection reduces the value of being careful today. We call this effect the fatalism effect. The planner, on the other hand, internalizes the possibility of re-infection and a congested healthcare system and as a result, her incentives to avoid infection increase sharply when she learns of the disease.

Our quantitative results show that this leads to significant differences in the evolution of both macro and health outcomes. In equilibrium, the representative household's response closely tracks the path of the infection rate. At the peak, labor supply drops by about $10 \%$ and as many as $60 \%$ of work happening from home. The result is a time path for infections that peaks at the same time as the zero-mitigation timepath albeit at a lower level. At the peak, the fataility rate is triple the baseline level. The planner, on the other hand, acts more quickly and aggressively to flatten the curve, cutting labor supply by $20 \%$ and prescribing a work-from-home ratio of $80 \%$ at the height of the disease. The infection rate peaks occurs a few weeks later and at a much lower level - almost half - that of the equilibrium level. The peak fatality rate and the cumulative fatalities are also significantly reduced. The ability to work from home plays a significant role in ameliorating the economic impact of the mitigation strategies. While this is true both in the case of private agents as well as the planner, the latter uses this optiom much more intensively. If we were to abstract from this option, the planner's optimal strategy has a peak drop in consumption of almost $45 \%$ (compared to $30 \%$ in the baseline model).

We then examine the robustness of our main results to assumptions about parameters 
(notably the fatality rate) and the arrival of news about the disease. We also consider a version with altruistic households and find that the time path of mitigation remains relatively unchanged for modest degrees of altruism.

Literature. There are by now a number of papers studying the tradeoff between economic and health outcomes in the context of the Covid-19 pandemic. It is impossible to cite every paper from this body of work, so we restrict ourselves to closely related early ones - Barro et al. (2020), Eichenbaum et al. (2020) and Alvarez et al. (2020). Barro et al. (2020) and Correia et al. (2020) draw lessons from the 1918 flu epidemic. Barro et al. (2020) find a high death rate (about 40 million people, $2 \%$ of the population at the time) and a large but not extreme impact on the economy (cumulative loss in GDP per capita of $6 \%$ over 3 years). The impact on the stock market was small. Correia et al. (2020) find that early interventions help protect health and economic outcomes. Our paper relates to an older literature on contagion dynamics, e.g. (Diekmann and Heesterbeek, 2000). We refer to the reader to Atkeson (2020b) for a recent discussion. Berger et al. (2020) show that testing can reduce the economic cost of mitigation policies as well as reduce the congestion in the health care system. Baker et al. (2020) document the early consumption response of households in the US.

Our model shares with Eichenbaum et al. (2020) the idea of embedding $S I R$ dynamics in a simple DSGE model. The SIR model is the same, but there are some notable differences in the economic model. Eichenbaum et al. (2020) consider hand-to-mouth households while we use a representative household framework in the tradition of Lucas and Stokey (1987), where both consumption and health risks are pooled. Apart from this difference, we emphasize the role of learning-by-doing in working from home which adds an important dynamic choice. The planner invests in the new technology to mitigate future disruptions. We also highlight the dynamic tension (the so-calledfatalism bias) between the planner and the private sector's incentives. 


\section{Benchmark Model}

\subsection{Households}

There is a continuum of mass $N$ of households. Each household is of size 1 and the utility of the household is

$$
U=\sum_{t=0}^{\infty} \beta^{t} u\left(c_{t}, l_{t} ; i_{t}, d_{t}\right)
$$

where $c_{t}$ and $l_{t}$ are per-capita consumption and labor supply. The household starts with a continuum of mass 1 of family members, all of them susceptible to the disease. At any time $t>0$ we denote by $s_{t}, i_{t}$ and $d_{t}$ the numbers of susceptible, infected and dead people. The size of the household at time $t$ is therefore $1-d_{t}$ and total household consumption is $\left(1-d_{t}\right) c_{t}$. Among the $i_{t}$ infected members, $\kappa i_{t}$ are too sick to work. The labor force at time $t$ is therefore $1-d_{t}-\kappa i_{t}$, and household labor supply is $\left(1-d_{t}-\kappa i_{t}\right) l_{t}$. The number of household members who have recovered from the disease is $r_{t}=1-s_{t}-i_{t}-d_{t}$. In our quantitative analysis, we use the functional form

$$
u\left(c_{t}, l_{t} ; i_{t}, d_{t}\right)=\left(1-d_{t}-\kappa i_{t}\right)\left(\log \left(c_{t}\right)-\frac{l_{t}^{1+\eta}}{1+\eta}\right)+\kappa i_{t}\left(\log \left(c_{t}\right)-u_{\kappa}\right)-u_{d} d_{t}
$$

where $u_{\kappa}$ is the disutility from being sick and $u_{d}$ the disutility from death which includes lost consumption and the psychological cost on surviving members. ${ }^{1}$ For simplicity, we assume that sickness does not change the marginal utility from consumption. This implies that the household will equate consumption for all alive members (i.e. independent of health status). The variables $s_{t}, i_{t}$ and $d_{t}$ evolve according to a standard $S I R$ model described below.

At the beginning of time $t$, each household decides how much to consume $c_{t}$ (per capita) and how much each able-bodied member should work $l_{t}$. We have normalized the disutility of labor so that $l=c=1$ before the epidemic starts. Households become infected by shopping and by going to work. We compute infection in two steps. First, we define exposure levels for shoppers and for workers. Then, we aggregate these into a composite infection rate

\footnotetext{
${ }^{1}$ Formally $u_{d}=$ PsyCost $-\log \left(c_{d}\right)$ where $c_{d}$ is the consumption equivalent in death. Technically we cannot set $c_{d}=0$ with $\log$ preferences but $u_{d}$ is a large number.
} 
at the household level. We assume throughout the paper that asymptomatic individuals are unaware of their infection. Formally, $\{$ dead $\}$ and $\{$ sick $\}$ are the only observable states at the individual level. In particular, households cannot tell the difference between the $s_{t}$ members who are susceptible and the $(1-\kappa) i_{t}$ members who are infected but not sick. This modeling choice is the main difference between our model and the model of Eichenbaum et al. (2020) who make the polar assumption. They assume that individuals know their types, do not share risks within a family, and behave in a hand-to-mouth fashion. We follow instead a Lucas and Stokey (1987) approach to model households' decisions and risk sharing, so our model stays close to a standard representative agent model.

We take into account the possibility of a cure/vaccine by adjusting the discount factor. Formally, this is equivalent to assuming that a cure/vaccine arrives stochastically at an exogenous arrival rate. Note that this combines both a treatment - which instantly cures sick individuals - and a vaccine - which eliminates the risk of future infections. Under this assumption, the economy jumps back to $l_{t}=c_{t}=1$ when the cure/vaccine arrives. We can therefore focus on the path along which a cure has not yet been found, along with a suitable adjustment to the discount factor. Let $\tilde{\beta}$ be the pure time discount rate and $\nu$ the likelihood of a vaccine. We define the effective discount factor $\beta=\tilde{\beta}(1-\nu)$ along the no-cure path.

\subsection{Shopping}

We define consumption exposure as

$$
e^{c} c_{t} C_{t}
$$

where $e^{c}$ measures the sensitivity of exposure to consumption and $C_{t}$ is aggregate consumption. $^{2}$ The idea behind this specification is that household members engage in various activities related to consumption - such as shopping in a crowded mall, eating inside a restaurant, going to a hospital - which exposes them to infection risk. These are assumed to proportional to consumption expenditure $c_{t}$ and for a given level of aggregate consumption, exposure is proportional to such activities. We study heterogeneity across sectors later

\footnotetext{
${ }^{2}$ We use lower-case letters to denote household level variables and upper-case for aggregates.
} 
in the paper.

\subsection{Production and Working from Home}

Production uses only labor, but a key feature of our model is the distinction between hours supplied by able bodied workers $l_{t}$ and effective labor supply $\hat{l}_{t}$ per household. Effective labor supply is

$$
\hat{l}_{t}=\left(1-d_{t}-\kappa i_{t}\right)\left(l_{t}-\frac{\chi_{t}}{2} m_{t}^{2}\right)
$$

The first term captures the fact that the number of valid household member is decreased by death and sickness. The second term captures the cost of implementing mitigation strategies, denoted $m_{t}$ (e.g., working from home at least some of the time). These strategies come at a cost - lost productivity, denoted by the term $\frac{\chi_{t}}{2} m_{t}^{2}$. The loss process $\chi_{t}$ is a decreasing function of the accumulated experience working from home and is given by:

$$
\chi_{t}=\chi\left(\bar{m}_{t}\right)
$$

where $\bar{m}_{t}$ is the stock of accumulated mitigation (with a depreciation rate of $1-\rho_{m}$ )

$$
\bar{m}_{t+1}=\rho_{m} \bar{m}_{t}+m_{t}
$$

The function $\chi$ is positive, decreasing, and convex. In the quantitative analysis, we assume the following functional form

$$
\chi_{t}=\bar{\chi}\left(1-\Delta_{\chi}\left(1-\exp \left(-\bar{m}_{t}\right)\right)\right)
$$

The cost shifter initially (i.e. when $\bar{m}_{t}=0$ ) is equal $\bar{\chi}>0$ and then falls over time as people figure out how to work from home. The parameter $\Delta_{\chi}$ indexes the maximum potential for learning by doing, since as $\bar{m}_{t}$ becomes large, the cost approaches $\bar{\chi}\left(1-\Delta_{\chi}\right)$.

The benefit of mitigation strategies is a reduction in the risk of infection. Specifically, 
exposure from work is given by

$$
e^{l}\left(1-m_{t}\right) l_{t}\left(1-M_{t}\right) L_{t}
$$

where, as before, upper-case letters denote aggregates.

The aggregate resource constraint is

$$
Y_{t}=C_{t}=\hat{L}_{t}=N \hat{l}_{t}
$$

In our basic model, we ignore the issue of firm heterogeneity and market power. Therefore, price is equal to marginal cost

$$
P_{t}=W_{t}=1
$$

where $W_{t}$ is the wage per unit of effective labor, which we normalize to one.

\subsection{Income and Contagion}

At the end of each period, household members regroup and share income and consumption. Household labor income is $W_{t} \hat{l}_{t}=\hat{l}_{t}$ and the budget constraint is

$$
\left(1-d_{t}\right) c_{t}+\frac{b_{t+1}}{1+r_{t}} \leq b_{t}+\hat{l}_{t}
$$

Household exposure is

$$
e_{t}=\bar{e}+\left(1-d_{t}\right) e^{c} c_{t} C_{t}+\left(1-d_{t}-\kappa i_{t}\right) e^{l}\left(1-m_{t}\right) l_{t}\left(1-M_{t}\right) L_{t}
$$


where $\bar{e}$ is baseline exposure, independent of market activities. Contagion dynamics follow a modified $S I R$ model (the benchmark model is explained in the appendix):

$$
\begin{aligned}
s_{t+1} & =s_{t}-\gamma e_{t} \frac{I_{t}}{N} s_{t} \\
i_{t+1} & =\gamma e_{t} \frac{I_{t}}{N} s_{t}+(1-\rho) i_{t}-\delta_{t} \kappa i_{t} \\
d_{t+1} & =d_{t}+\delta_{t} \kappa i_{t} \\
r_{t+1} & =r_{t}+\rho i_{t}
\end{aligned}
$$

where $\gamma$ is the infection rate per unit of exposure, $\rho$ the recovery rate, $\kappa$ the probability of being sick conditional on infection, and $\delta_{t}$ the mortality rate of sick patients. In the standard $S I R$ model, exposure is assumed to be constant, whereas here it depends on consumption, labor supply and mitigation strategies. Note that the number of new infected, $\gamma e_{t} \frac{I_{t}}{N} s_{t}$, is a function of the aggregate infection rate, taken as given by households.

Finally, the mortality rate $\delta_{t}$ is described by an increasing function of the measure of sick agents:

$$
\delta_{t}=\delta\left(\kappa I_{t}\right)
$$

which captures the idea that an overloaded healthcare system can contribute to higher fatalities.

\subsection{Market Clearing and Aggregate Dynamics}

Infection dynamics for the the entire population are simply given by the $S I R$ system above with aggregate variable $I_{t}=N i_{t}$, and so on. The aggregate labor force is $N\left(1-\kappa i_{t}-d_{t}\right) l_{t}$ and total consumption is $N\left(1-d_{t}\right) c_{t}$. The market clearing conditions are

$$
\left(1-d_{t}\right) c_{t}=\hat{l}_{t}
$$

and for the bond market

$$
b_{t}=0
$$




\section{Decentralized equilibrium}

\subsection{Equilibrium Conditions}

Since our model reduces to a representative household model and since $b=0$ in equilibrium, we simply omit $b$ from the value function. The household's optimal choices are the solution to the following recursive problem:

$$
V\left(i_{t}, s_{t}, d_{t}, \bar{m}_{t}\right)=\max _{c_{t}, l_{t}, m_{t}} u\left(c_{t}, l_{t} ; i_{t}, d_{t}\right)+\beta V\left(i_{t+1}, d_{t+1}, s_{t+1}, \bar{m}_{t+1}\right)
$$

where $\bar{m}_{t+1}=\bar{m}_{t}+m_{t}$ and

$$
u\left(c_{t}, l_{t} ; i_{t}, d_{t}\right)=\left(1-d_{t}\right) \log \left(c_{t}\right)-\left(1-d_{t}-\kappa i_{t}\right) \frac{l_{t}^{1+\eta}}{1+\eta}-u_{\kappa} \kappa i_{t}-u_{d} d_{t}
$$

with effective labor given by

$$
\begin{aligned}
\hat{l}_{t} & =\left(1-d_{t}-\kappa i_{t}\right)\left(l_{t}-\frac{\chi_{t}}{2} m_{t}^{2}\right) \\
\chi_{t} & =\bar{\chi}\left(1-\Delta_{\chi}\left(1-\exp \left(-\bar{m}_{t}\right)\right)\right)
\end{aligned}
$$

The Lagrangian is then:

$$
\begin{aligned}
V_{t} & =u\left(c_{t}, l_{t} ; i_{t}, d_{t}\right)+\beta V_{t+1}+\lambda_{t}\left(\hat{l}_{t}+b_{t}-\left(1-d_{t}\right) c_{t}-\frac{b_{t+1}}{1+r_{t}}\right) \\
& +\lambda_{e, t}\left(e_{t}-\bar{e}-\left(1-d_{t}\right) e^{c} c_{t} C_{t}-\left(1-d_{t}-\kappa i_{t}\right) e^{l}\left(1-m_{t}\right) l_{t}\left(1-M_{t}\right) L_{t}\right) \\
& +\lambda_{i, t}\left(i_{t+1}-\gamma e_{t} \frac{I_{t}}{N} s_{t}-(1-\rho) i_{t}+\delta_{t} \kappa i_{t}\right) \\
& +\lambda_{s, t}\left(s_{t+1}-s_{t}+\gamma e_{t} \frac{I_{t}}{N} s_{t}\right) \\
& +\lambda_{d, t}\left(d_{t+1}-d_{t}-\delta_{t} \kappa i_{t}\right)
\end{aligned}
$$

The first order conditions for consumption and labor are then 


$$
\begin{aligned}
c_{t} & : c_{t}^{-1}=\lambda_{t}+\lambda_{e, t} e^{c} C_{t} \\
l_{t} & : l_{t}^{\eta}=\lambda_{t}-\lambda_{e, t} e^{l}\left(1-m_{t}\right)\left(1-M_{t}\right) L_{t} \\
m_{t}: & \lambda_{t} \chi_{t} m_{t}=\frac{\beta V_{\bar{m}, t+1}}{1-d_{t}-\kappa i_{t}}+\lambda_{e, t} e^{l} l_{t}\left(1-M_{t}\right) L_{t}
\end{aligned}
$$

The remaining first order conditions are

$$
\begin{aligned}
e_{t}: \lambda_{e, t} & =\left(\lambda_{i, t}-\lambda_{s, t}\right) \gamma \frac{I_{t}}{N} s_{t} \\
i_{t+1}: \lambda_{i, t} & =-\beta V_{i, t+1} \\
s_{t+1}: \lambda_{s, t} & =-\beta V_{s, t+1} \\
d_{t+1}: \lambda_{d, t} & =-\beta V_{d, t+1}
\end{aligned}
$$

The envelope conditions are

$$
\begin{aligned}
V_{i, t} & =\kappa \frac{l_{t}^{1+\eta}}{1+\eta}-\kappa u_{\kappa}-\kappa \lambda_{t}\left(l_{t}-\frac{\chi_{t} m_{t}^{2}}{2}\right)+\lambda_{e, t} \kappa e^{l}\left(1-m_{t}\right) l_{t}\left(1-M_{t}\right) L_{t}-(1-\rho) \lambda_{i, t}+\delta_{t} \kappa\left(\lambda_{i, t}-\lambda_{d, t}\right) \\
V_{s, t} & =\left(\lambda_{s, t}-\lambda_{i, t}\right) \gamma e_{t} \frac{I_{t}}{N}-\lambda_{s, t} \\
V_{d, t} & =\frac{l_{t}^{1+\eta}}{1+\eta}-\log \left(c_{t}\right)-u_{d}-\lambda_{t}\left(l_{t}-\chi_{t} m_{t}^{2}-c_{t}\right)+\lambda_{e, t}\left(e^{c} c_{t} C_{t}+e^{l}\left(1-m_{t}\right) l_{t}\left(1-M_{t}\right) L_{t}\right)-\lambda_{d, t} \\
V_{\bar{m}, t} & =\beta V_{\bar{m}, t+1}+\lambda_{t}\left(1-d_{t}-\kappa i_{t}\right) \frac{\bar{\chi}}{2} m_{t}^{2} \Delta_{\chi} \exp \left(-\bar{m}_{t}\right)
\end{aligned}
$$

\subsection{Equilibrium with Exogenous Infections}

To simplify the notation, we normalize $N=1$, so we should think of our values as being per-capita pre-infection. When there is no risk of contagion, i.e. $i_{t}=0, \lambda_{e, t}=0$ and $V_{\bar{m}, t+1}=0$, we have $m_{t}=0$ and from the optimal consumption and labor supply

$$
c_{t}^{-1}=l_{t}^{\eta}
$$


Since $m=0$ we have $\hat{l}_{t}=l_{t}$ so market clearing is simply

$$
c_{t}=l_{t}
$$

Combining these two conditions we get

$$
c_{t}=l_{t}=1
$$

The pre-infection economy is always in steady state.

Consider now an economy with exogenous SIR dynamics: $e^{c}=e^{l}=0$. This implies $m_{t}=0$ and

$$
c_{t}^{-1}=l_{t}^{\eta}
$$

Market clearing requires

$$
\left(1-d_{t}\right) c_{t}=\left(1-d_{t}-\kappa i_{t}\right) l_{t}
$$

therefore

$$
l_{t}^{1+\eta}=1+\frac{\kappa i_{t}}{1-d_{t}-\kappa i_{t}}
$$

The labor supply of valid workers increases to compensate for the reduced productivity of the sick. Per capita consumption is

$$
c_{t}=\left(\frac{1-d_{t}}{1-d_{t}-\kappa i_{t}}\right)^{-\frac{\eta}{1+\eta}}
$$

As long as $\eta>0$ consumption per capita decreases. Aggregate GDP decreases because of lost labor productivity and deaths.

The $S I R$ system is independent from the economic equilibrium. As described in the Appendix, the share of infected agents $I_{t}$ increases, reaches a maximum and converges to 0 in the long run. Assuming a constant $\delta$, the long run solution solves

$$
\log \left(\frac{S_{\infty}}{1-I_{0}}\right)=-\frac{\gamma \bar{e}}{\rho+\delta \kappa}\left(\frac{1-S_{\infty}}{N}\right)
$$


and

$$
D_{\infty}=\frac{\delta \kappa}{\delta \kappa+\rho}\left(1-S_{\infty}\right)
$$

When the congestion externality arises and $\delta_{t}$ increases, then we cannot obtain a closedform solution for the long run death rate but the qualitative results are unchanged. The following proposition summarizes our results.

Proposition 1. When contagion does not depend on economic activity $\left(e^{c}=e^{l}=0\right)$, the share of infected agents $I_{t}$ increases, reaches a maximum and converges to 0 in the long run. The long run death rate is given by $D_{\infty}=\frac{\delta \kappa}{\delta \kappa+\rho}\left(1-S_{\infty}\right)$ where the long run share of uninfected agents solves $\log \left(\frac{S_{\infty}}{1-I_{0}}\right)=-\frac{\gamma \bar{e}}{\rho+\delta \kappa}\left(\frac{1-S_{\infty}}{N}\right)$. Along the transition path, labor supply of able-bodied workers follows the infection rate while per-capita consumption moves in the opposite direction as $c_{t}=\left(1-\frac{\kappa i_{t}}{1-d_{t}}\right)^{\frac{\eta}{1+\eta}}$.

\subsection{Private Incentives for Mitigation}

In this subsection, we characterize incentives of households to undertake mitigation. The incentives to mitigate depend on the shadow value of exposure $\lambda_{e, t}=\left(\lambda_{i, t}-\lambda_{s, t}\right) \gamma \frac{I_{t}}{N} s_{t}$ which is increasing in new infections, $\gamma \frac{I_{t}}{N} s_{t}$. So, for a given $\lambda_{i, t}-\lambda_{s, t}$, the private incentives to mitigate are proportional to the number of new cases. Now,

$$
\lambda_{i, t}-\lambda_{s, t}=\beta\left(V_{s, t+1}-V_{i, t+1}\right)
$$

with

$$
\begin{aligned}
V_{s, t}-V_{i, t} & =u_{\kappa} \kappa+\kappa l_{t}\left(\lambda_{t}-\frac{l_{t}^{\eta}}{1+\eta}\right)-\rho \lambda_{i, t}+\left(1-\gamma e_{t} \frac{I_{t}}{N}\right)\left(\lambda_{i, t}-\lambda_{s, t}\right)+\delta_{t} \kappa\left(\lambda_{d, t}-\lambda_{i, t}\right) \\
& =u_{\kappa} \kappa+\kappa l_{t}\left(\lambda_{t}-\frac{l_{t}^{\eta}}{1+\eta}\right)-\rho \lambda_{i, t}+\left(1-\gamma e_{t} \frac{I_{t}}{N}\right) \beta\left(V_{s, t+1}-V_{i, t+1}\right)+\delta_{t} \kappa \beta\left(V_{i, t+1}-V_{d, t+1}\right)
\end{aligned}
$$

Fatalism Effect We now use an approximation to obtain a sharper characterization and gain more intuition. Specifically, we make the following assumptions (i) the non-monetary cost of death $u_{d}$ is sufficiently large that it dominates the other terms in the expression for $V_{d, t}$ and $V_{i, t}$ (ii) there are no congestion effects on fatality, i.e. $\delta_{t}=\delta$. Then, the value of 
death and infection are approximately constant, i.e.

$$
\begin{aligned}
& V_{d, t} \approx-\frac{u_{d}}{1-\beta} \\
& V_{i, t} \approx \sum_{s=0}^{\infty} \beta^{s}(1-\rho-\delta \kappa)^{s} \kappa\left(-u_{\kappa}+\delta \frac{\beta u_{d}}{1-\beta}\right)=\frac{\kappa u_{\kappa}+\delta \kappa \frac{\beta}{1-\beta} u_{d}}{1-\beta(1-\rho-\delta \kappa)} \equiv V_{i}
\end{aligned}
$$

The value of avoiding an infection at time $t$ is the discounted value of the disutility from sickness and death. The value of remaining susceptible is then

$$
V_{s, t} \approx V_{i} \sum_{s=1}^{\infty} \beta^{s}\left(1-\gamma e_{t+s-1} \frac{I_{t+s-1}}{N}\right)^{s-1} \gamma e_{t+s-1} \frac{I_{t+s-1}}{N}
$$

Importantly, $V_{s, t}$ falls with the risk of infection in the near future. Formally,

$$
\begin{aligned}
\frac{\partial V_{s, t}}{\partial \frac{I_{t+s-1}}{N}} & =V_{i} \beta^{s}\left[\left(1-\gamma e_{t+s-1} \frac{I_{t+s-1}}{N}\right)^{s-1} \gamma e_{t+s-1}-(s-1)\left(1-\gamma e_{t+s-1} \frac{I_{t+s-1}}{N}\right)^{s-2}\left(\gamma e_{t+s-1}\right)^{2} \frac{I_{t+s-1}}{N}\right] \\
& =V_{i} \beta^{s}\left(1-\gamma e_{t+s-1} \frac{I_{t+s-1}}{N}\right)^{s-1} \gamma e_{t+s-1}\left[1-(s-1) \frac{\gamma e_{t+s-1} \frac{I_{t+s-1}}{N}}{1-\gamma e_{t+s-1} \frac{I_{t+s-1}}{N}}\right]
\end{aligned}
$$

The term inside the square bracket is positive (or eq., $\frac{\partial V_{s, t}}{\partial \frac{I_{t+s-1}}{N}}$ is negative) for low $s$. Since $V_{i, t}$ is approximately constant, this means that the difference $V_{s, t}-V_{i, t}$ shrinks when agents become aware of the disease, i.e. there is a perverse effect on incentives to mitigate. We term this channel the fatalism effect: early on, agents (correctly) anticipate that they are likely to become infected and so have weak incentives to avoid infection today. As we will see, the planner's solution considers other forces that offset this channel.

When $\delta$ is constant the fatalism effect rationally reduces privates incentives to mitigate the disease, i.e., $V_{s, t}-V_{i, t}$ is small, but it does not switch the sign, i.e., $V_{s, t}-V_{i, t}$ remains positive. This can change when $\delta$ is time varying as we discuss later.

\section{Planner's Problem}

Again, we normalize $N=1$ for simplicity. The planner solves 


$$
\max U=\sum_{t=0}^{\infty} \beta^{t} u\left(C_{t}, L_{t} ; I_{t}, D_{t}\right)
$$

subject to

$$
u\left(C_{t}, L_{t} ; I_{t}, D_{t}\right)=\left(1-D_{t}\right) \log \left(C_{t}\right)-\left(1-D_{t}-\kappa I_{t}\right) \frac{L_{t}^{1+\eta}}{1+\eta}-u_{\kappa} \kappa I_{t}-u_{d} D_{t}
$$

and

$$
\left(1-D_{t}\right) C_{t}=\left(1-D_{t}-\kappa I_{t}\right)\left(L_{t}-\frac{\chi_{t}}{2}\left(M_{t}\right)^{2}\right)
$$

The first order conditions for consumption and labor are then (highlighted in red are the differences with the decentralized equilibrium)

$$
\begin{aligned}
& C_{t}: \quad C_{t}^{-1}=\lambda_{t}+2 \lambda_{e, t} e^{c} C_{t} \\
& L_{t}: \quad L_{t}^{\eta}=\lambda_{t}-2 \lambda_{e, t} e^{l}\left(1-M_{t}\right)^{2} L_{t} \\
& M_{t}: \quad \lambda_{t} \chi_{t} M_{t}=\frac{\beta V_{\bar{M}, t+1}}{1-D_{t}-\kappa I_{t}}+2 \lambda_{e, t} e^{l}\left(1-M_{t}\right) L_{t}^{2}
\end{aligned}
$$

The marginal utilities of the planner with respect to exposure are twice as high as those of the private sector because of the contagion externalities: private agents only care about how their behavior affect their own infection risk. They do not care about how their behavior affects the infection risk of others.

The envelope condition that changes (relative to the equilibrium) is the one with respect to the number of infected people:

$$
\begin{gathered}
V_{I, t}=\kappa \frac{L_{t}^{1+\eta}}{1+\eta}-\kappa u_{\kappa}-\kappa \lambda_{t}\left(L_{t}-\chi_{t} M_{t}^{2}\right)+\lambda_{e, t} \kappa e^{l}\left(1-M_{t}\right)^{2} L_{t}^{2}-(1-\rho) \lambda_{I, t} \\
-\gamma e_{t} S_{t}\left(\lambda_{I, t}-\lambda_{S, t}\right)-\left(\delta_{t} \kappa+\delta_{t}^{\prime} \kappa^{2} I_{t}\right)\left(\lambda_{D, t}-\lambda_{I, t}\right)
\end{gathered}
$$

where $\delta_{t}^{\prime}$ denotes the derivative of the fatality rate with respect to the sick population. Let us now consider the planner's incentives to reduce consumption today in response to . 


\subsection{Incentives to Mitigate}

Next, we characterize the planner's mitigation incentives and contrast them with the private incentives studied in section 3.3. The planner's incentives to avoid infection today depend on $V_{S, t+1}-V_{I, t+1}$. Under the same simplifying assumptions as before, $V_{D, t}$ is approximately constant (and the same as that of private agents):

$$
V_{D} \approx-\frac{u_{d}}{1-\beta}
$$

while the values of being infected and remaining susceptible are

$$
\begin{aligned}
& V_{I, t} \approx \sum_{s=0}^{\infty} \beta^{s}\left(\prod_{\tau=0}^{s-1}\left(1-\rho+\gamma e_{t+\tau} S_{t+\tau}-\delta \kappa\right)\right)\left(\kappa u_{\kappa}+\delta \kappa \frac{\beta}{1-\beta} u_{d}\right) \\
& V_{S, t}=\sum_{s=1}^{\infty} \beta^{s}\left(1-\gamma e_{t+s-1} \frac{I_{t+s-1}}{N}\right)^{s-1} V_{I, t+s} \gamma e_{t+s-1} \frac{I_{t+s-1}}{N}
\end{aligned}
$$

The expression for $V_{S, t}$ shows that the planner also suffers from the fatalism effect: risk of future infection reduces the value of remaining safe today. But there is an additional effect which arises because of the $\gamma e_{t} S_{t}$ term in the first equation. This implies that the value of infection $V_{I, t}$ is amplified (i.e. becomes even more negative) when the planner becomes aware of the disease. Intuitively, the possibility of spreading makes the effective recovery rate from the planner's point of view equal to $\rho-\gamma e_{t} S_{t}$ is instead of $\rho$.

Thus, from the planner's point of view, both $V_{I, t}$ and $V_{S, t}$ become more negative when the disease arrives. The net effect, which drives her incentives to mitigate, depend on the relative strength of the two forces. Early on, the risk of an infected agent spreading the disease to others is quite high (since $S_{t}$ is high) while the fatalism effect (which depends on $\left.\frac{I_{t+\tau}}{N}\right)$ is relatively muted, and therefore the incentives to mitigate are particularly powerful.

Finally, while we abstracted from congestion effects in this discussion for simplicity, reintroducing them creates additional incentives for the the planner to mitigate. To see why, note that with congestion effects, the planner's value of infection is given by (the terms that 
change relative to the case without congestion are highlighted in red):

$V_{I, t}=\sum_{s=0}^{\infty} \beta^{s}\left(\prod_{\tau=0}^{s-1}\left(1-\rho+\gamma e_{t+\tau} S_{t+\tau}-\delta_{t+\tau} \kappa\right)\right)\left(\kappa u_{\kappa}+\delta_{t+s} \kappa \frac{\beta}{1-\beta} u_{d}+\delta_{t+s}^{\prime} \kappa^{2} I_{t+s} \frac{\beta}{1-\beta} u_{d}\right)$

Thus, the planner hates infection even more in the presence of congestion effects. This externality introduces another wedge between the private and social incentives to mitigate. This tension helps explain episodes like the Florida spring break controversy. In March 2020, as the arrival of the pandemic became broadly known in the US, many people chose to enjoy their spring break, arguing that if they were going to catch the virus, now would be as good a time as later, while public officials worried about the spread of the disease and hospitals being overwhelmed.

\section{Calibration}

Before detailing our calibration strategy, we note that considerable uncertainty about key parameters remains even now. For an early discussion of the challenges, see Atkeson (2020a). We return to this issue later in the paper. We calibrate our model at the weekly frequency.

Contagion The SIR block of the model is parameterized as follows. The recovery parameter is set to $\rho=0.35$ following Atkeson (2020b). This value implies that the average duration for which an infected agent is contagious is about 20 days. The fraction of infected people who fall sick is $\kappa=0.15$. For the exposure function, we normalize $\bar{e}+e^{c}+e^{l}=1$ and set $e^{c}=e^{l}=\frac{1}{3}$, consistent with the estimates in Ferguson (2020). These parameters imply that total exposure $e=1$ at the pre-pandemic levels of consumption and labor (the calibration of production and utility parameters will be described later). The parameter $\gamma$ is then chosen to target the basic reproduction number (i.e. the average number of people infected by a single infected individual) of $\mathcal{R}=3.3$ consistent with the estimate of Salje et al. (2020), yielding an estimated value of $\gamma=1.2$. Finally, to parameterize the fatality rate and the congestion effects, we adopt the following functional form for the case fatality rate $\delta_{t}:$

$$
\delta_{t}=\bar{\delta}+\exp \left(\phi I_{t}\right)-1
$$


where the parameter $\phi$ indexes the strength of the congestion externality. We set $\bar{\delta}$ and $\phi$ to match two targets for the case fatality rate: a baseline value (i.e. the fraction of infected people who die even in the absence of congestion) of $0.5 \%$ and an 'extreme' value of $1.5 \%$ when $3 \%$ of the population requires medical attention (i.e. the fraction of people who die when $\kappa I=0.15 \times 0.2)$. This procedure yields $\bar{\delta}=0.012$ and $\phi=0.8$. We examine the robustness of our results to these choices later in the paper.

Preferences and technology The utility parameter $u_{d}$ is set to a baseline value of 10 , implying a flow disutility from death that is 10 times per capita income. Such large nonmonetary costs associated with loss of life are consistent with estimates in the literature and with values used by government entities like the EPA. For example, Greenstone and Nigam (2020) use an estimated value of a statistical life of $\$ 11.5$ million (in 2020 dollars) to the household from death. Assuming a rate of return of 5\%, this translates into an annual flow value of $\$ 575,000$, or roughly 10 times per capita GDP. The flow disutility from sickness $u_{\kappa}$ is set to a value of 0.5 .

As we discussed earlier, our discount factor $\beta$ is meant to captures both time discounting and the possibility of a cure/vaccine arriving at an constant, exogenous rate. The pure time discounting component corresponds to an annual discount factor of 0.95 . For the cure/vaccine, we take a relatively pessimistic case as our baseline, with the annualized probability of $15 \%$. The composite annual discount factor thus becomes 0.8 , or equivalently, a weekly $\beta=(0.8)^{\frac{1}{52}}=0.9957$.

Next, we calibrate the working-from-home technology, which involves picking values for two parameters $-\bar{\chi}$ and $\Delta_{\chi}$. For the former, we use estimates of the effect of the restrictions imposed by many countries in February and March 2020. A back-of-the-envelope calculation suggests that a policy which requires almost the entire labor force to work from home (i.e. $m \approx 1$ ) with no prior experience $(\bar{m} \approx 0)$ causes GDP to fall by $25 \%$ below its normal level. Since this initial productivity loss is exactly equal to $\frac{\bar{\chi}}{2}$, we set $\bar{\chi}=0.5$. Finally, for the long-run parameter, $\Delta_{\chi}$, we rely on Dingel and Neiman (2020), who estimate that roughly one-third of the jobs in the US can be done from home. Guided by this estimate, we set $\Delta_{\chi}=0.34$. We also assume that the cumulation of learning depreciates 
very slowly, i.e. $\rho_{m}=0.99$, which implies that the stock of knowledge evolves according to $\bar{m}_{t+1}=0.99 \bar{m}_{t}+m_{t}$.

\section{Quantitative Results}

Our benchmark exercise uses a large initial infection rate of $i_{0}=1 \%$ for expositional purposes (it makes the figures easier to read in the early periods), but it is important to keep in mind that this is a large shock. It seems likely that agents and policy makers become aware of the epidemic much earlier, so in our robustness analysis, we also report simulations starting at $i_{0}=0.1 \%$.

Private Response We start with the decentralized solution. Figure 1 and Figure 2 show the behavior of the contagion and macro variables in the decentralized equilibrium, under three different assumptions about exposure and mitigation strategies. The blue line solid shows a situation where infection rates are exogenous, i.e. do not vary with the level of economic activity. Since infection is assumed to be exogenous, agents do not engage in mitigation, i.e., they ignore the pandemic. In fact, labor input rises (the solid line, top left panel in Figure 2), while per-capita consumption falls by about 2.5\% (the dashed line), as able-bodied workers work harder to compensate for the workers who are sick. This is clear not a realistic assumption, but it serves as a useful benchmark for the worst case scenario. In this scenario, eventually almost the entire population is infected and $1.75 \%$ of the population succumbs to the virus (bottom, left panel in Figure 1). The case mortality rate peaks at $2.5 \%$ roughly 15 weeks after the initial infection when about $6 \%$ (15\% of the peak infection rate of $40 \%$ ) of the population is sick and the healthcare system is overwhelmed.

The red line describes the case where exposure is endogenous (i.e. varies with consumption and labor) but there is no work-from-home (WFH) technology, i.e, the only ways for the household to reduce exposure are to cut back on its consumption and labor supply. As we would expect, this leads to a sharp reduction in economic activity (top, left panel in Figure 2) by almost 30\%. Importantly, the reduction is gradual, tracking the overall infection rate (consumption and labor hit their trough at 17 weeks for ). Intuitively, when the 
Figure 1: Decentralized Equilibrium: Contagion Dynamics
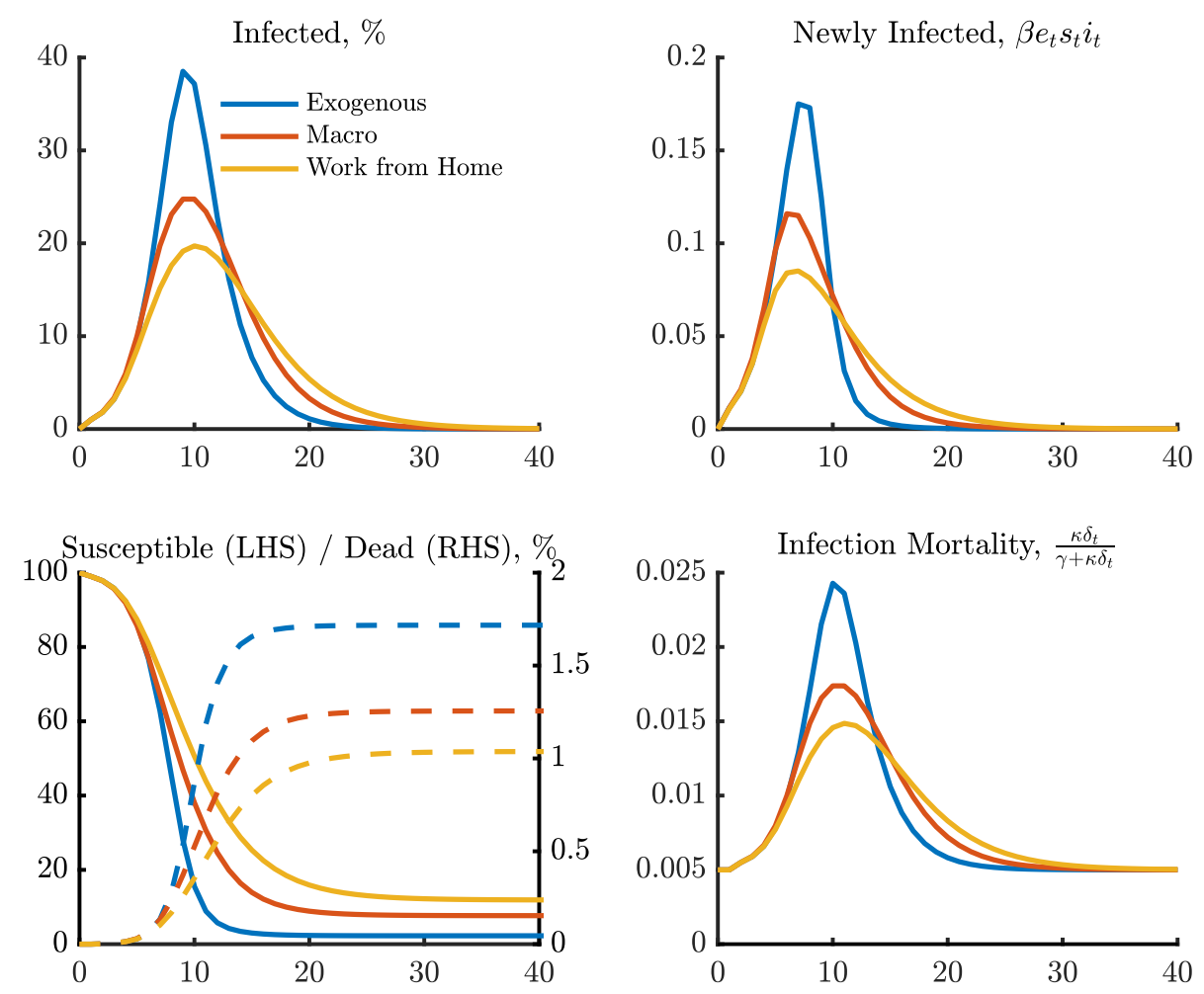

fraction of infected people is low (as is the case in the early stages), a reduction in exposure has a small effect on future infection risk, relative to the resulting fall in consumption. And since each household does not internalize the effect it has on the future infection rate, it has little incentive to indulge in costly mitigation early on. This dynamic is reflected in the hump-shaped pattern in $\lambda_{e}$ (the bottom, middle panel in Figure 2). As we will see, the planner's incentives change much more strongly at the beginning. The mitigation behavior does lower the cumulative infection and death rates (relative to the exogenous infection case), by about $5 \%$ and $0.5 \%$ respectively.

Finally, the yellow line shows the effect of access to the WFH technology. This allows the household to reduce exposure without sacrificing consumption - now, the peak loss in consumption is $20 \%$ (compared to $30 \%$ without the WFH option), even as the exposure falls by more (0.55 compared to 0.65 , bottom left panel in Figure 2). The fraction of time spent working from home (top, left panel in Figure 2) is hump-shaped, peaking at $60 \%$ at roughly the same time as the fraction of infected rate. This additional flexibility also lowers cumulative fatalities to $1 \%$. However, the timing of mitigation strategies is mostly 
Figure 2: Decentralized Equilibrium: Aggregates
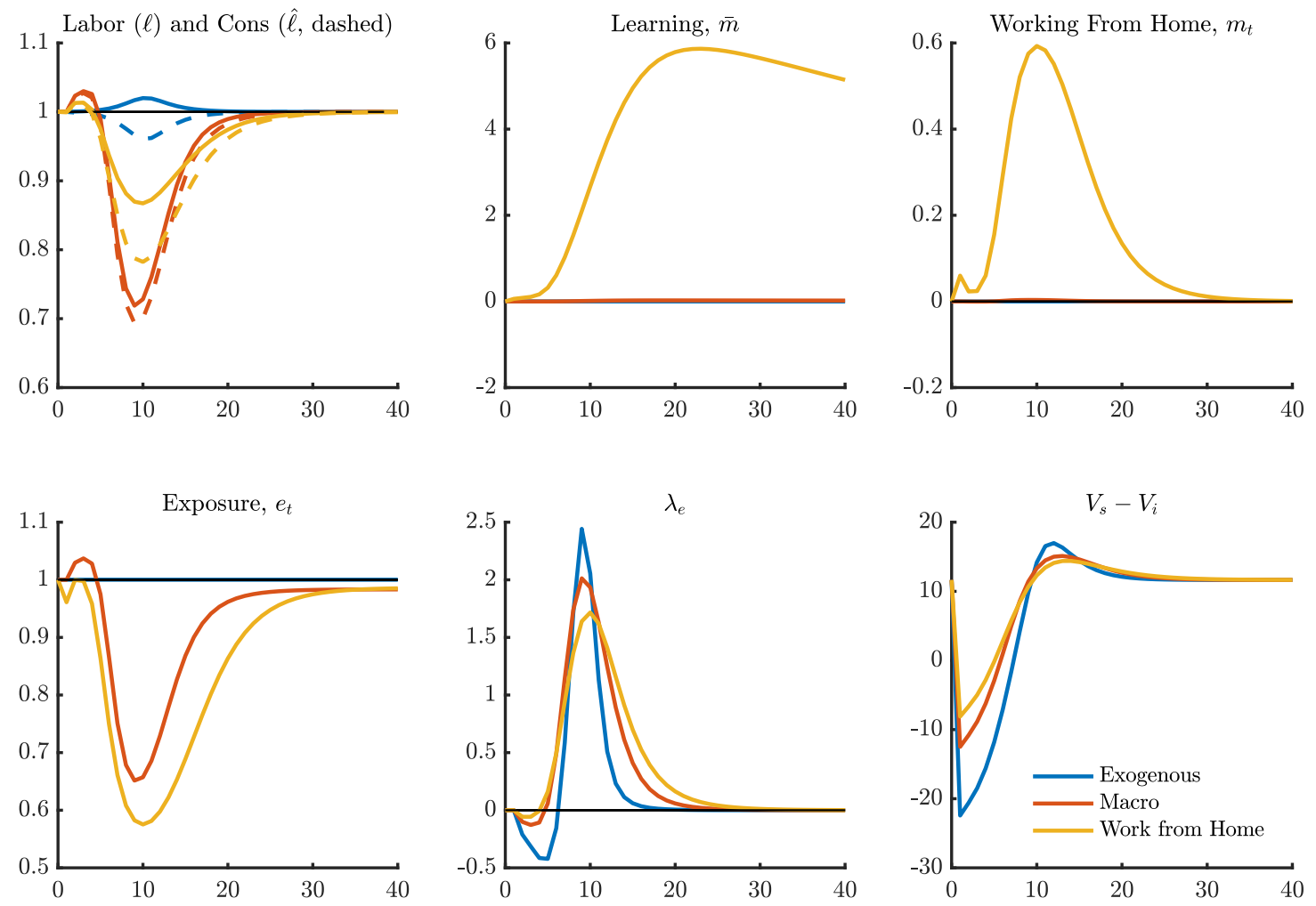

unchanged - households in the decentralized economy do not find it optimal to front-load their mitigation efforts.

Optimal Response We now turn to the planner's solution, depicted in Figures 3 and 4. As before, the blue, red and yellow lines show the cases of exogenous infection, mitigation without WFH and mitigation with WFH respectively. As the yellow and red curves in Figure 3 show, the planner finds it optimal to "flatten the curve" more dramatically than agents in equilibrium. The peak infection rates are well below the decentralized equilibrium levels (10\% versus $20 \%$ ), leading to a lower peak fatality rate (1\% versus $1.5 \%$ ) as well as cumulative deaths $(0.7 \%$, compared to slightly over $1 \%$ in the decentralized equilibrium with WFH). To achieve this, the planner has to cut by more than $50 \%$. Of course, this pushes the economy into a deeper recession with consumption falling by more than $30 \%$ even with WFH (top left panel in Figure4). The planner's response also displays a humpshaped pattern, rising with the infection rate, but she does step on the brakes sooner (almost immediately) compared to private agents. 
Figure 3: Planner Solution: Contagion Dynamics
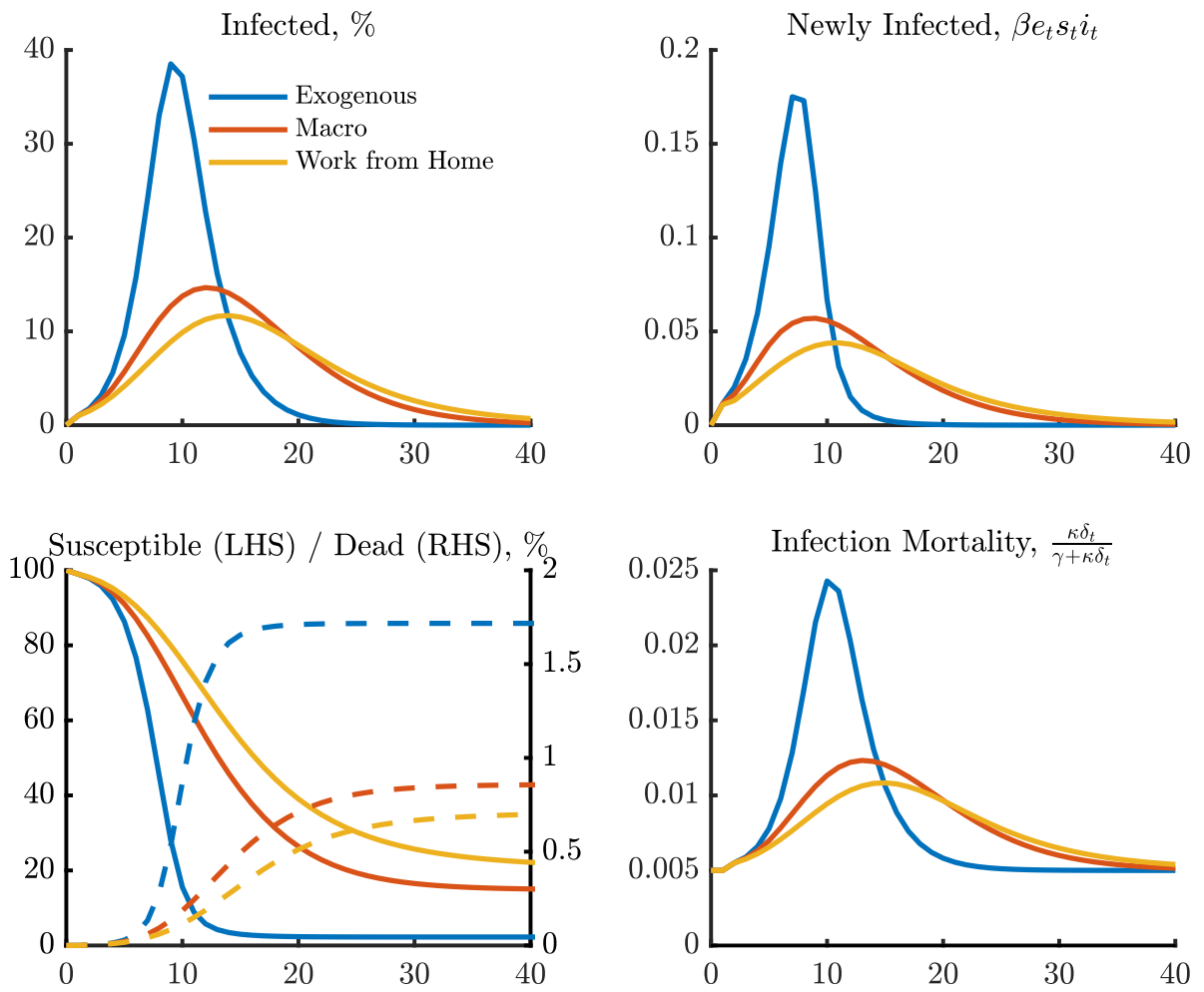

Figure 4: Planner Solution: Aggregates
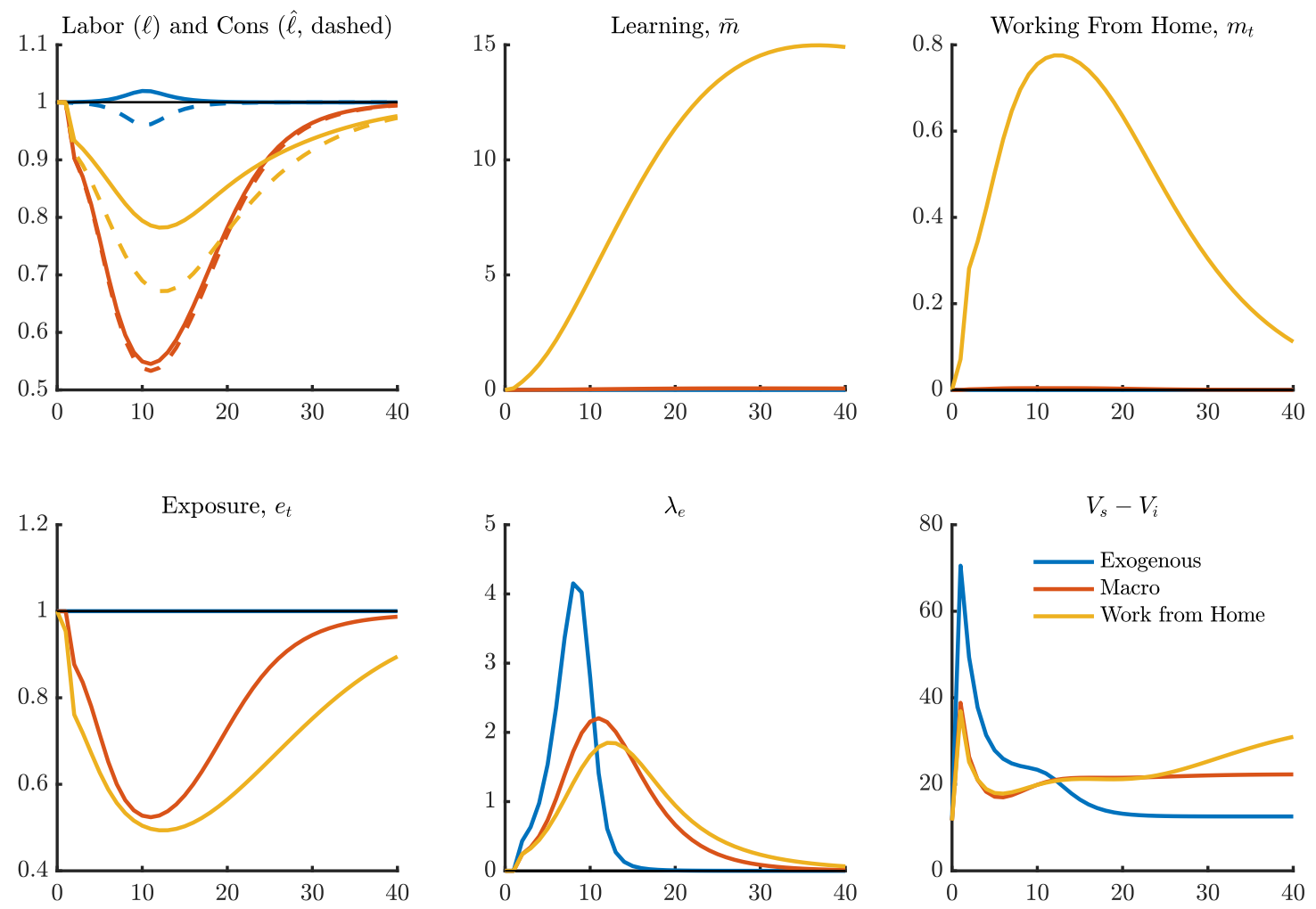
The availability of the WFH technology ameliorates the economic impact of the planner's suppression strategy, but does not significantly alter the contagion dynamics. Intuitively, fatalities are so costly that the planner aggressively suppresses the infection even in the absence of the WFH option: access to WFH simply allows the planner to achieve the same exposure outcomes at a lower cost. Notice also that the planner's incentives to use the WFH technology are much stronger than that of private agents in a decentralized equilibrium: partly because her shadow value of exposure is higher, but also because she attaches a greater value to the future benefits of accumulated knowledge. As a result, $m$ rises to as high as 0.8 relatively quickly. In other words, as long as a vaccine is not available, the planner has almost $80 \%$ of the population working from home at the peak of the disease.

Private versus Public Incentives Figure 5 illustrates the fatalism effect. The top graphs assume a constant risk of death $\delta$. On the left we see the shrinking private incentives for safety early on, as $V_{s, t}-V_{i, t}$ decreases. On the right, we see that $V_{s, t}-V_{i, t}$ increases for the planner. The lower graphs illustrate another incentive problem that arises when $\delta$ is time-varying. If agents anticipate congestion in the future and if they think they are likely to become infected, they might prefer to increase their risk of infection today because it is better to be sick when $\delta$ is still relatively low. Not only does $V_{s, t}-V_{i, t}$ shrink, its sign can even flip, as we see in the bottom left panel. For the planner, by contrast, the risk of a higher $\delta$ increases the incentives to mitigate. We conclude that the misalignment of private incentives is larger during early stages of the epidemic, and is amplified by congestion externalities.

Smaller initial shock What is the value of an early warning? Suppose the planner becomes aware of the disease at $i_{0}=0.1 \%$ (in the baseline, $i_{0}=1 \%$ ). Figures 6 and 7 show her optimal response in this case. The changes relative to the $i_{0}=1 \%$ case are relatively small - the extent of mitigation, work-from-home and the final death count are all quite similar across these cases (of course, as we would expect, the peaks occur slightly later with a smaller initial infection). These results suggest a modest value to detecting the disease at an earlier stage. 
Figure 5: Fatalism and Perverse Incentives
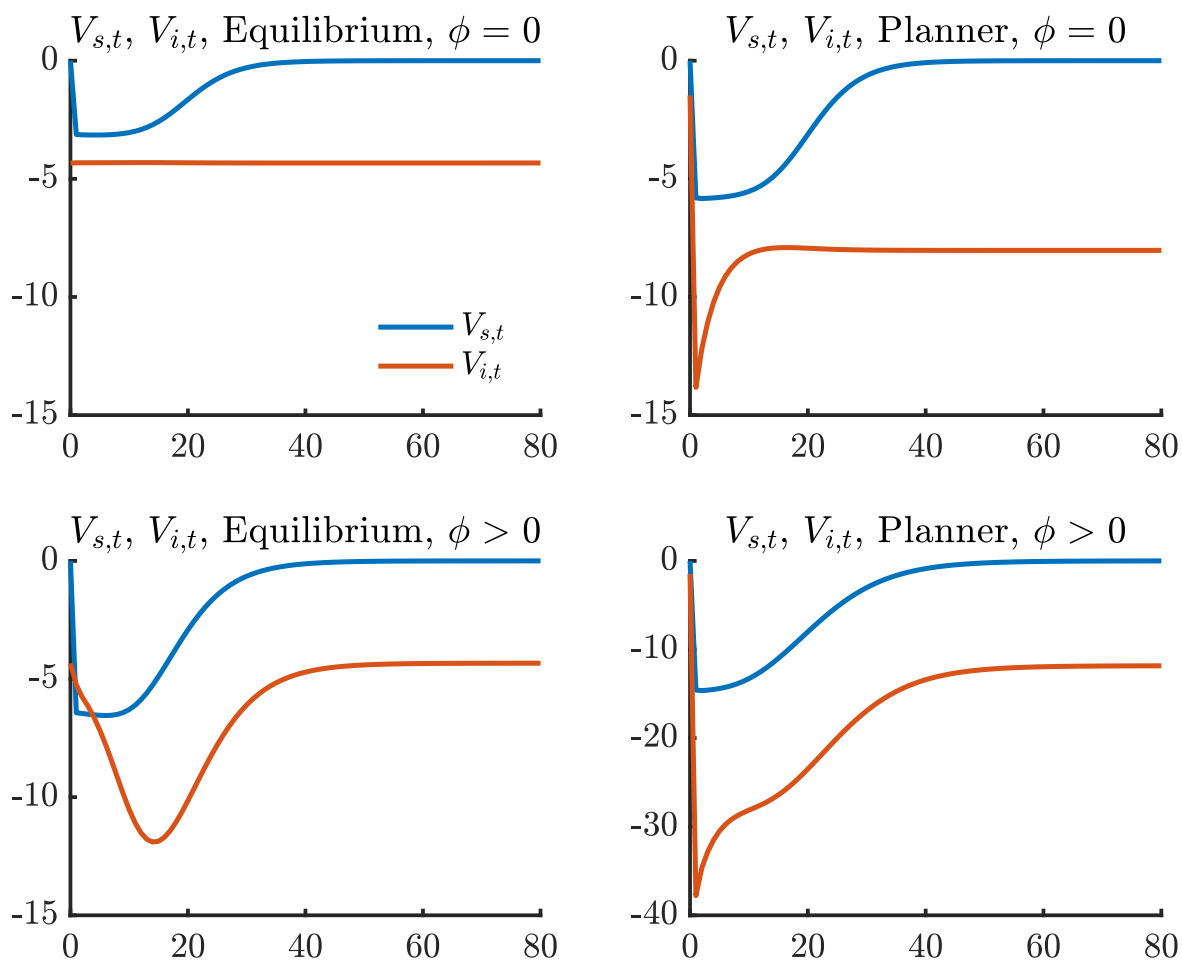

Figure 6: Planner Solution: Contagion Dynamics with Smaller Infection Shock
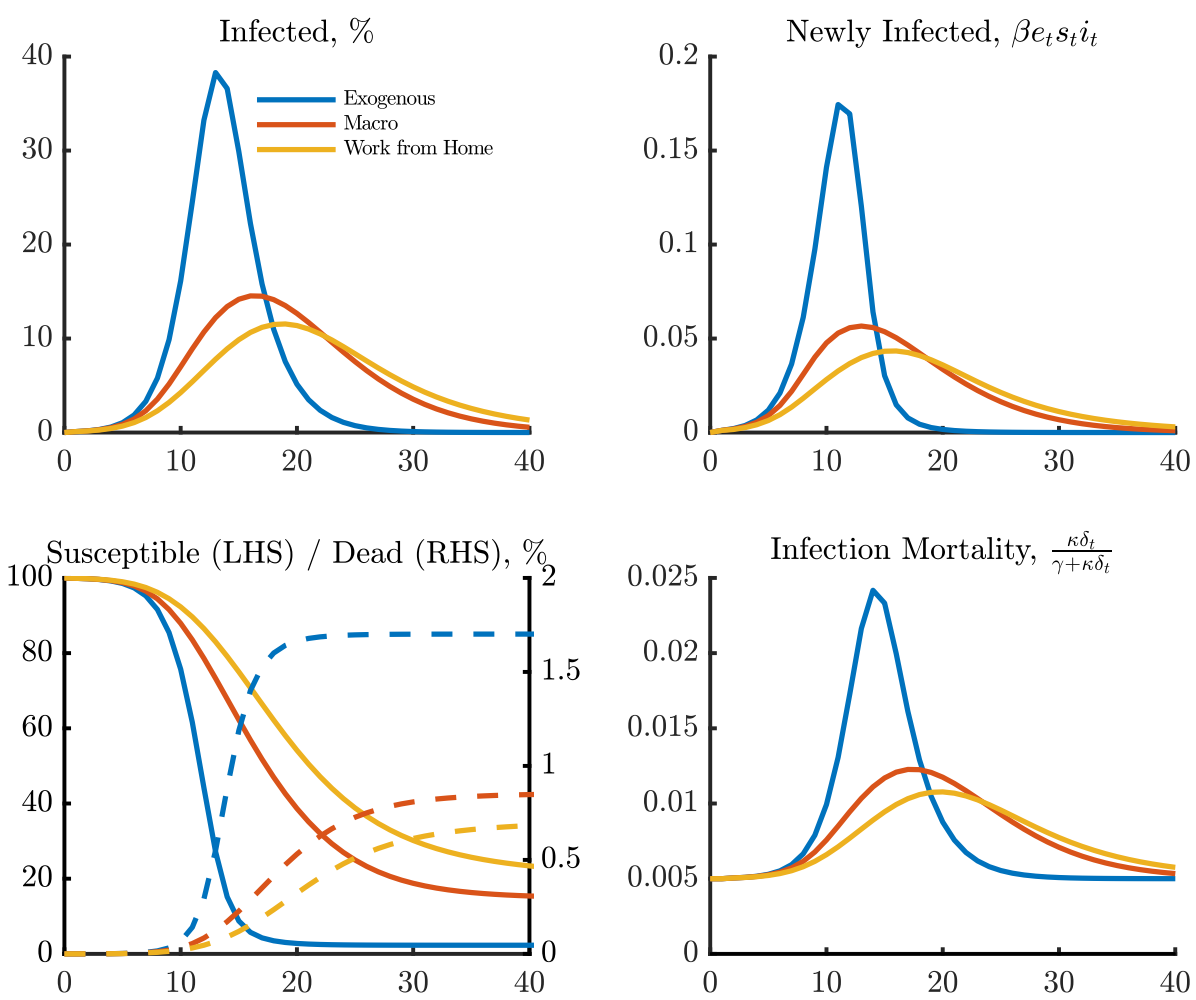
Figure 7: Planner Solution: Aggregates with Smaller Infection Shock
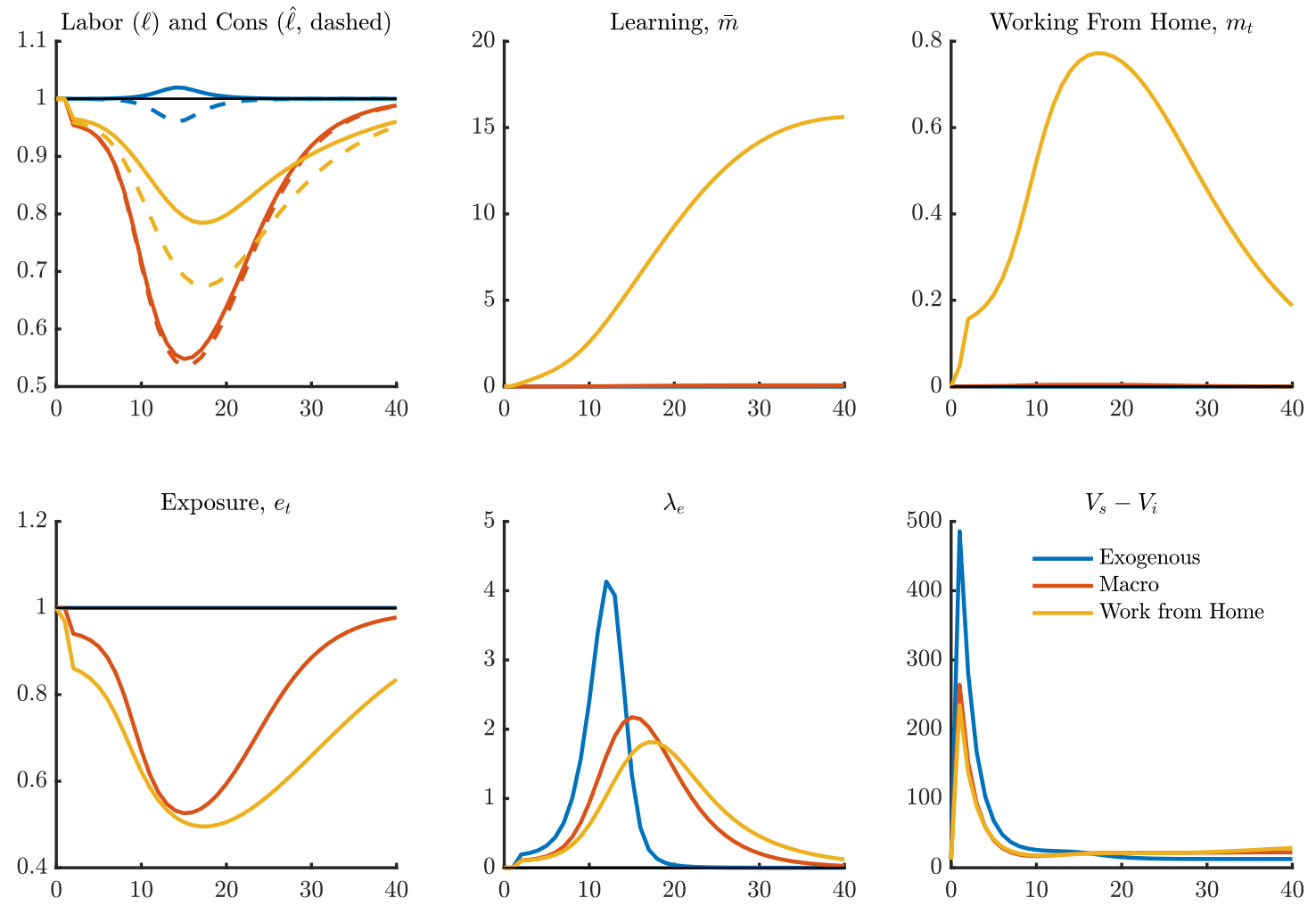

Lower fatality As we discussed earlier, there is little agreement in the literature on the mortality of Covid-19, with Atkeson (2020a) suggesting that the range of plausible values could be as wide as $0.1 \%$ to $1 \%$. To explore the effect of this uncertainty, we repeat our analysis of the planner's problem with mortality rates that are a tenth of our baseline values, i.e. probability of death (conditional on being) is now given by $\frac{1}{10} \delta\left(\kappa I_{t}\right)$. The results are presented in Figures 10 and 11. As expected, the overall number of fatalities are much lower, but the overall profile of the planner's solution is unchanged: she still finds it optimal to front load her intervention. The economic cost, however, is much lower and the lockdown finishes earlier to let the economy recover.

Congestion externality Next, to isolate the role of the congestion externality, we repeat our analysis of the planner's solution with the parameter $\phi$ set to 0 . The results, shown in Figures 10 and 11, display a similar pattern as Figures 3 and 4, but with a much less severe contraction. Intuitively, a healthcare system with sufficient slack capacity allows the planner to achieve similar outcomes in terms of fatalities with modestly higher infection 
Figure 8: Planner Solution: Contagion Dynamics with Reduced $\delta_{t}$
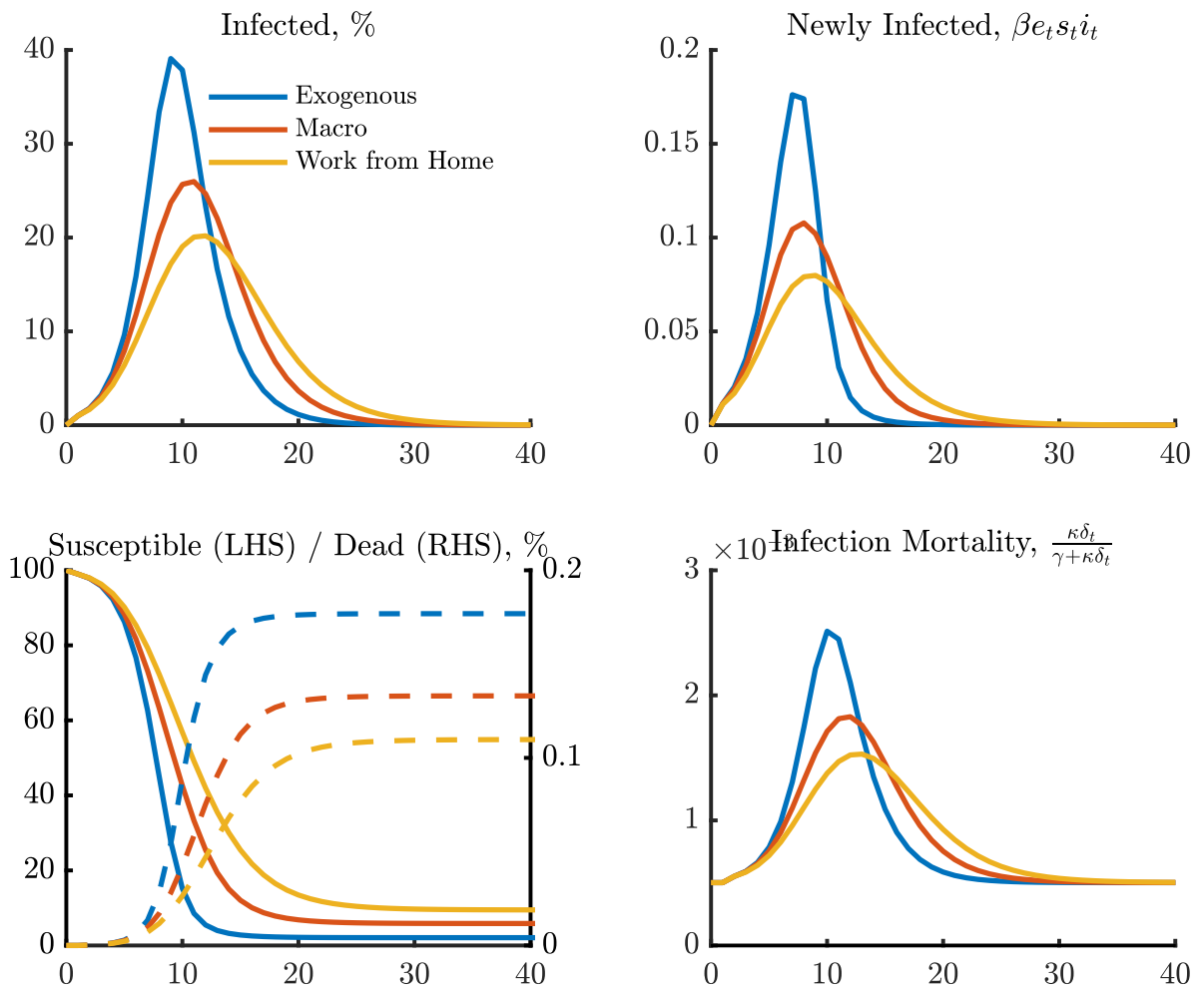

Figure 9: Planner Solution: Aggregates with Reduced $\delta_{t}$
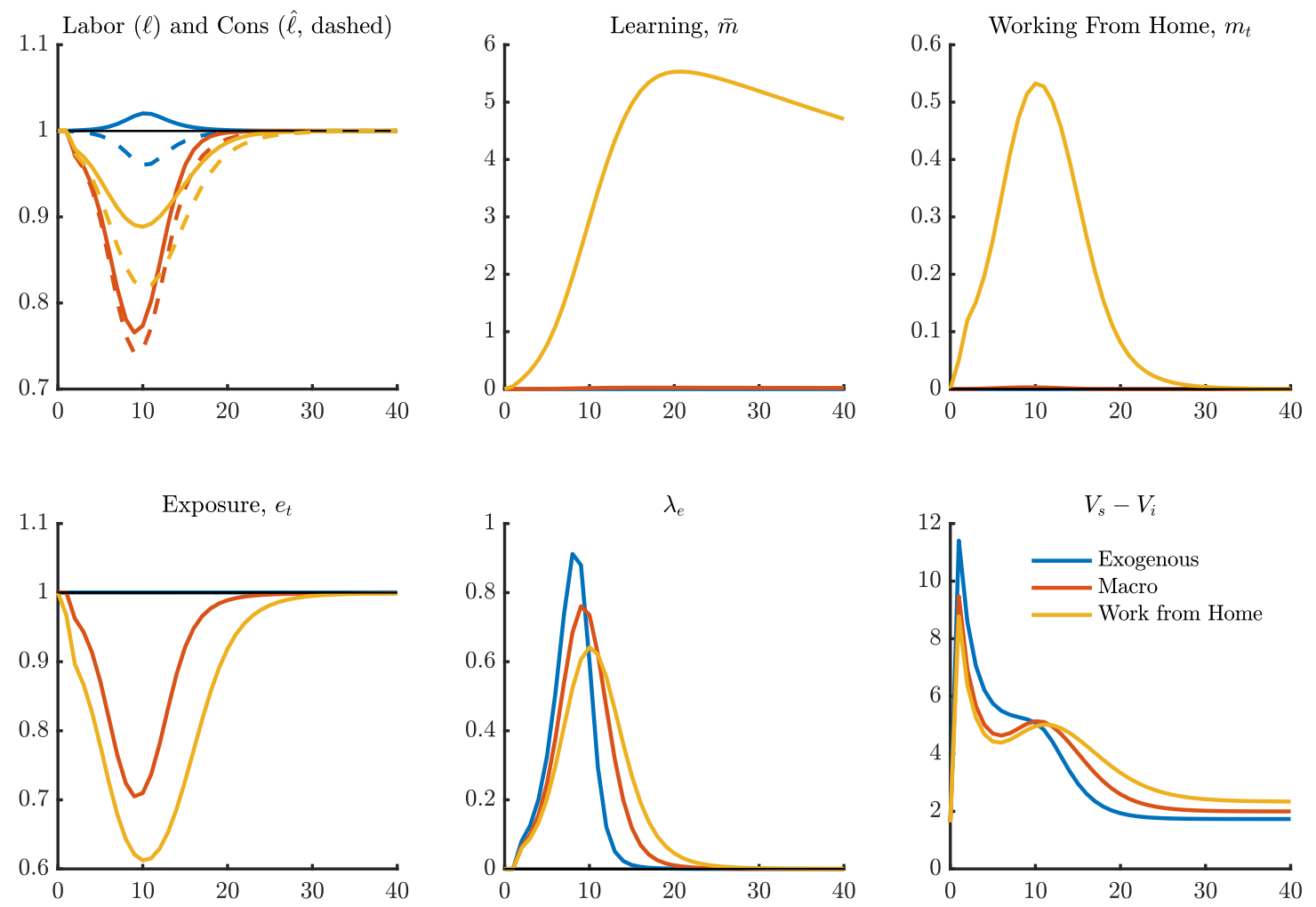
rates or equivalently, with less mitigation (top, left panel in Figure 11). Accordingly, the recession is not as deep or persistent than in the baseline.

Altruistic households Finally, we consider the possibility that households partly internalize their effects on the aggregate outcome. Formally, we assume that households incorporate externalities with a weight $\tau$ while making their consumption, labor and mitigation decisions. The laissez-faire equilibrium and the planner's optimum are obtained as special cases by setting $\tau=0$ and $\tau=1$ respectively. If $\tau=0.33$, the death rate is $0.9 \%$ (only slightly lower than the $1.0 \%$ under $\tau=0$ ). If $\tau$ is assumed to be 0.5 , i.e. households place equal weight on private and social incentives, the cumulative death rate falls to $0.85 \%$ (roughly a half of the gap between the laissez-faire equilibrium and the planner's optimum). These results show that for private decisions to be close to that of the planner requires a very large degree of altruism.

Parameter uncertainty As we briefly alluded to earlier, there was (and in some respects, and continues to be) much uncertainty about the structural parameters of the disease. Here, we use our model to speak to the dilemma faced by policy-makers who have to make decisions with imperfect knowledge of key primitives of the disease they are dealing with it. Atkeson (2020a) points out that, when one does not know the initial number of active cases, it is difficult "to distinguish whether the disease is deadly (1\% fatality rate) or milder $(0.1 \%$ fatality rate)." We illustrate this in Figure 12 where we show how two diseases with very different parameterizations can have similar implications for the number of dead, which is the variable that, in principle, is the easiest to measure. One disease has a reproduction number $\mathcal{R}=3.3$ and an infection fatality rate of $0.5 \%$, while the other disease has a lower reproduction number $\mathcal{R}=2.0$ but a higher infection fatality rate of $1.0 \%$. With an initial shock of $i_{0}=0.1 \%$, over the first two months of the spread of these diseases, the paths of the number of dead are almost identical, as shown in the first panel of the figure. Without accurate measures of other epidemiological variables, such as the number of newly infected or the fraction of cases recovered, it becomes nearly impossible to distinguish the two diseases in the short-term. Over the longer-term, however, the two diseases have very 
Figure 10: Planner Solution: Contagion Dynamics with $\phi=0$
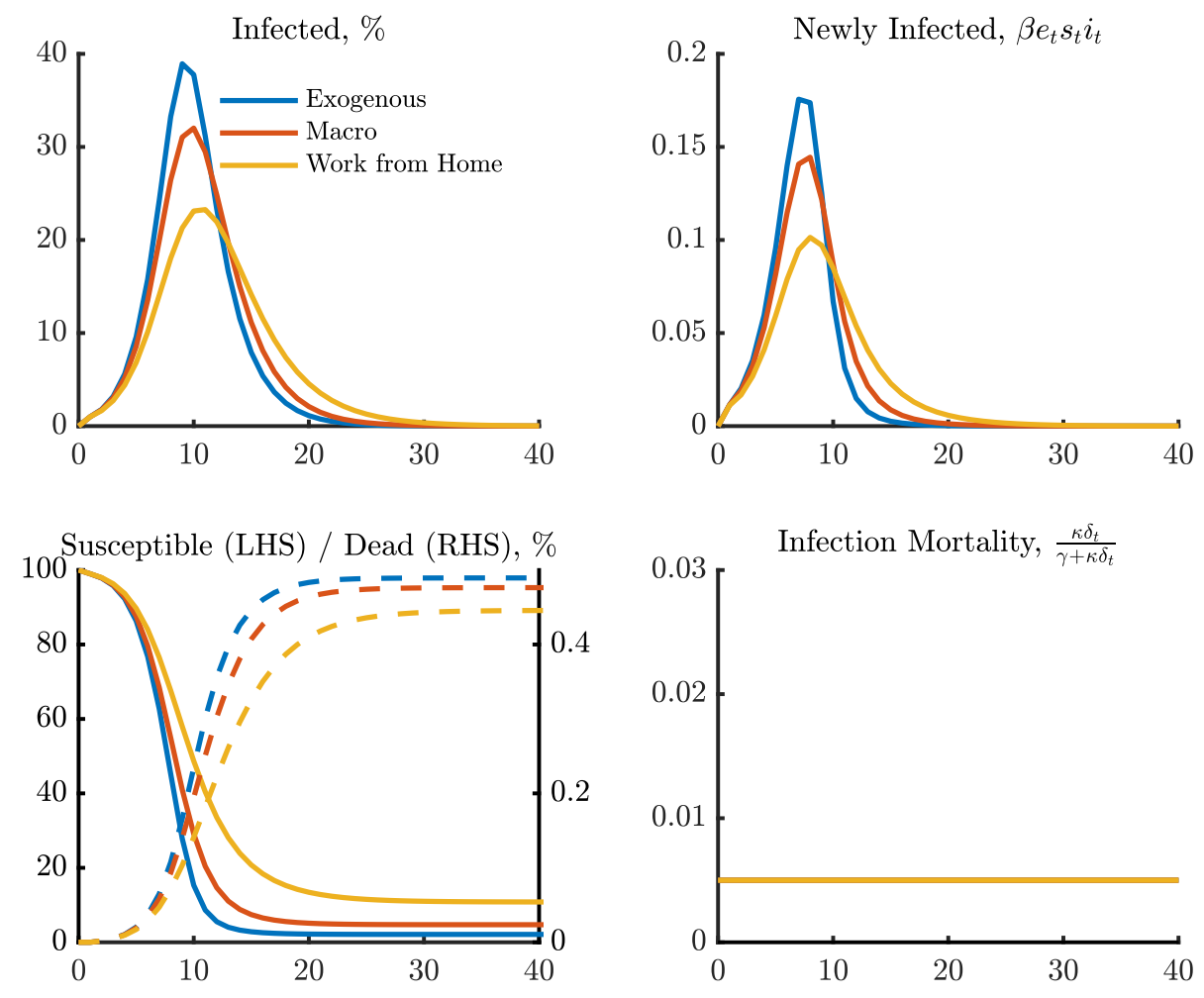

Figure 11: Planner Solution: Aggregates with $\phi=0$
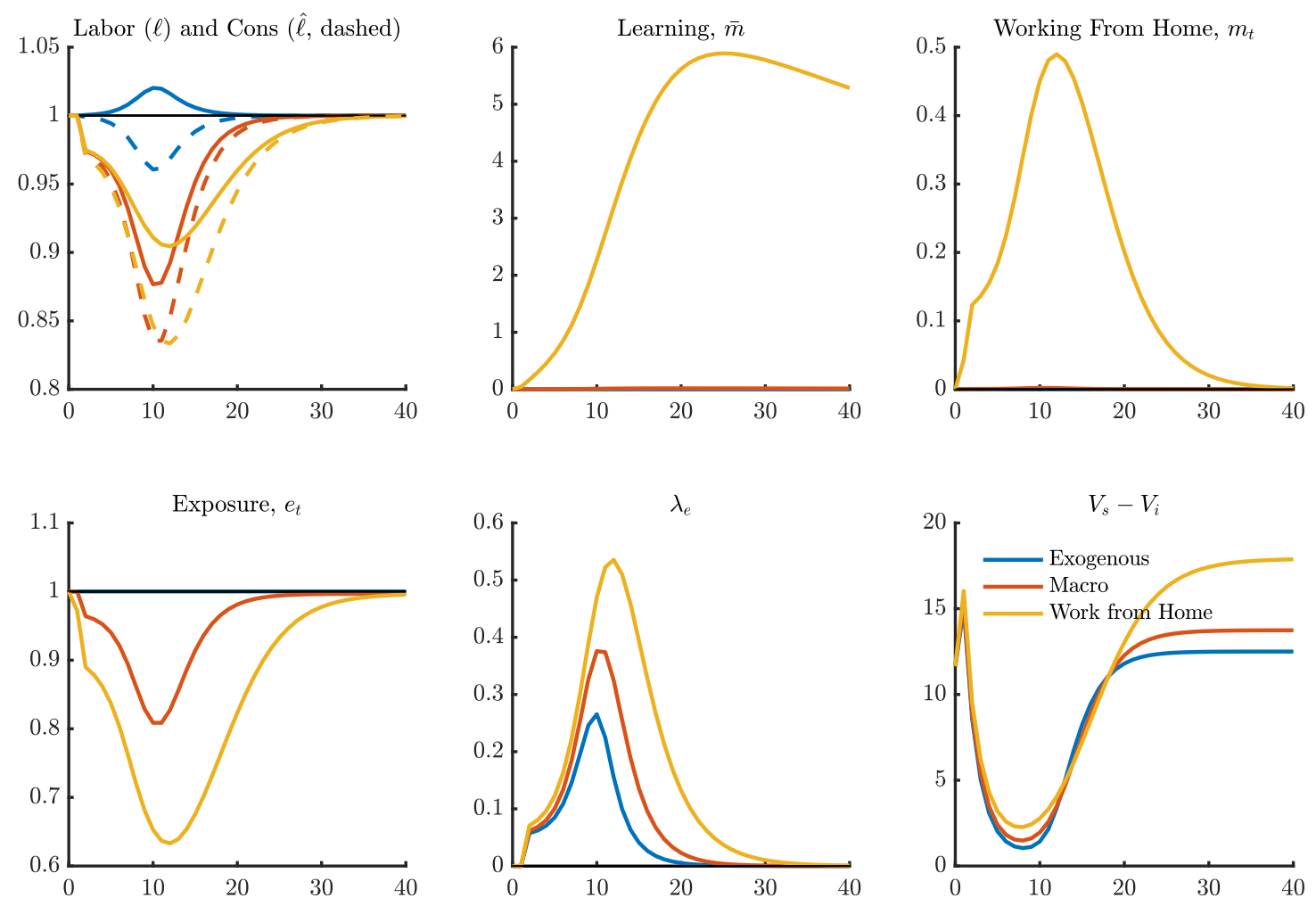
Figure 12: Percentage of Population Dead for Two Diseases and Time Horizons
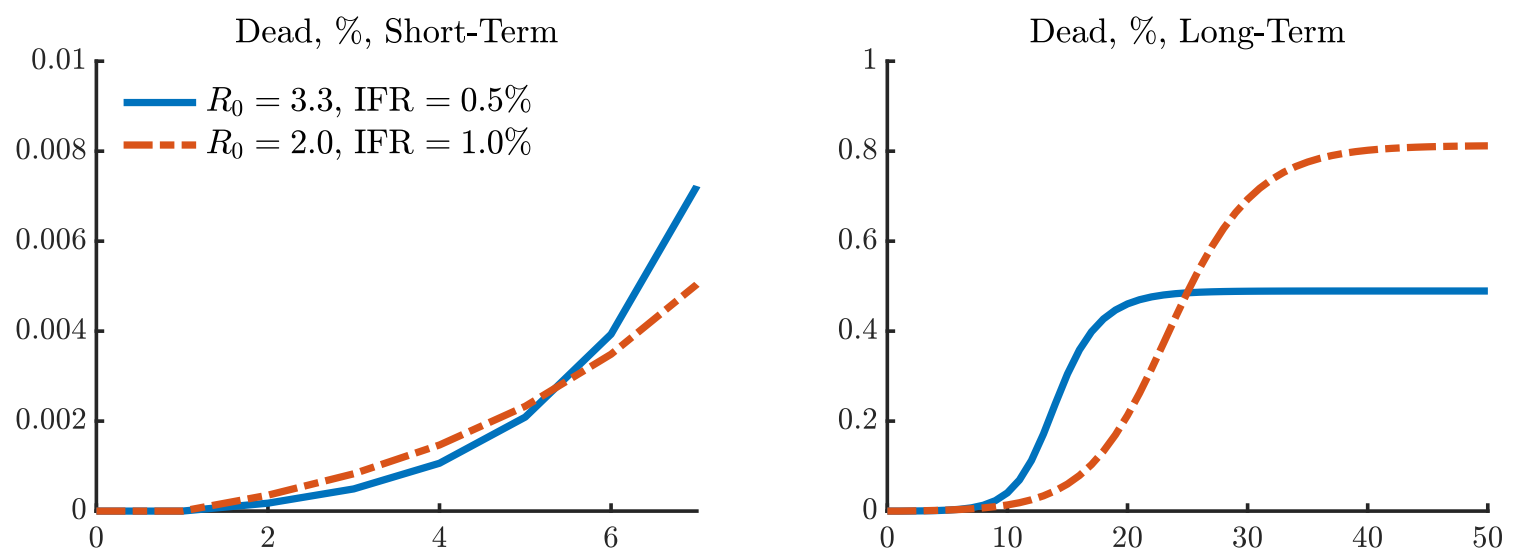

different implications for the number of dead, as shown in the second panel.

To explore more the role of uncertainty over the initial number of infected in our model under the baseline parameterization, we considered a deadly disease with a low initial infection rate of $i_{0}=0.1 \%$, and a milder disease with a high initial infection rate of $i_{0}=1 \%$. Interestingly, in both cases, the planner should implement immediately a strong suppression policy. The main difference is that in the mild case it is optimal to release the lockdown sooner. Thus, faced with large uncertainty about the fatality rate, the planner could impose a strong suppression policy and wait for more data. Assuming data available 20 weeks after the outbreak allows her to precisely estimate the fatality rates, this strategy would allow the planner to implement the optimal response, despite the initial uncertainty about a key parameter.

\section{Conclusions}

We propose an extension of the neoclassical model to include contagion dynamics, to study and quantify the tradeoffs of policies that can mitigate the Covid-19 pandemic. Our model reveals two key insights. First, that relative to the incentives of private agents, a planner wishes to significantly front-load mitigation strategies. Second, in our calibrated model, the prospect of mitigation together with the possibility of agents working from home gives quantitatively meaningful reductions in the spread of a disease and the economic costs.

Extensions of our baseline setup would yield a fruitful laboratory to study other policy- 
relevant questions. For instance, the production-side of the economy could be enriched to multiple and heterogeneous sectors, to provide additional margins to mitigate exposure and economic hardship. A richer model will also help to give sharper quantitative estimates on the costs of mitigation strategies. 


\section{References}

Alvarez, Fernando, David Argente, and Francesco Lippi, "A Simple Planning Problem for COVID-19 Lockdown," March 2020. Working Paper.

Atkeson, Andrew, "How Deadly is COVID-19? Understanding the Difficulties with Estimation," March 2020. NBER Working Paper No. 26867.

_, "What Will Be the Economic Impact of COVID-19 in the US? Rough Estimates of Disease Scenarios," March 2020. NBER Working Paper No. 26867.

Baker, Scott R., R.A. Farrokhnia, Steffen Meyer, Michaela Pagel, and Constantine Yannelis, "How Does Household Spending Respond to an Epidemic? Consumption During the 2020 COVID-19 Pandemic," University of Chicago, Working Paper, 2020.

Barro, Robert J, Jose Ursua, and Joanna Weng, "The coronavirus and the Great Influenza epidemic: Lessons from the "Spanish Flu" for the coronavirus' potential effects on mortality and economic activity," March 2020. AEI Economics Working Paper 202002.

Berger, David, Kyle Herkenhoff, and Simon Mongey, "An SEIR Infectious Disease Model with Testing and Conditional Quarantine," March 2020. Working Paper.

Correia, Sergio, Stephan Luck, and Emil Verner, "Pandemics Depress the Economy, Public Health Interventions Do Not: Evidence from the 1918 Flu," March 2020. Working Paper.

Diekmann, Odo and J. Heesterbeek, Mathematical Epidemiology of Infectious Diseases: Model Building, Analysis and Interpretation, John Wiley, 2000.

Dingel, Jonathan and Brent Neiman, "How Many Jobs Can be Done at Home?," March 2020. Working Paper.

Eichenbaum, Martin, Sergio Rebelo, and Mathias Trabandt, "The Macroeconomics of Epidemics," March 2020. Working Paper NWU. 
Ferguson, Neil, "Impact of non-pharmaceutical interventions (NPIs) to reduce COVID19 mortality and healthcare demand," March 2020. Imperial College Report.

Greenstone, Michael and Vishan Nigam, "Does Social Distancing Matter?," University of Chicago, Becker Friedman Institute for Economics Working Paper, 2020, (202026).

Harko, Tiberiu, Francisco Lobo, and M. K. Mak, "Exact analytical solutions of the Susceptible-Infected-Recovered (SIR) epidemic model and of the SIR model with equal death and birth rates," Applied Mathematics and Computation, 2014, (236), 184-194.

Lucas, Robert E. Jr. and Nancy L. Stokey, "Money and Interest in a Cash-in-Advance Economy," Econometrica,, 1987, 55 (3), 491-513.

Salje, Henrik, Cécile Tran Kiem, Noémie Lefrancq, Noémie Courtejoie, and Paolo Bosetti et al., "Estimating the burden of SARS-CoV-2 in France," 2020. Institut Pasteur. 


\section{Appendix}

\section{A Planner's Problem}

The planner understands that the dynamics follow

$$
\begin{aligned}
S_{t+1} & =S_{t}-\gamma e_{t} I_{t} S_{t} \\
I_{t+1} & =\gamma e_{t} I_{t} S_{t}+(1-\rho) I_{t}-\delta\left(\kappa I_{t}\right) \kappa I_{t} \\
D_{t+1} & =D_{t}+\delta\left(\kappa I_{t}\right) \kappa i_{t} \\
R_{t+1} & =R_{t}+\rho I_{t}
\end{aligned}
$$

We normalize $N=1$ for simplicity.

$$
\max U=\sum_{t=0}^{\infty} \beta^{t} u\left(C_{t}, L_{t} ; I_{t}, D_{t}\right)
$$

subject to

$$
u\left(C_{t}, L_{t} ; I_{t}, D_{t}\right)=\left(1-D_{t}\right) \log \left(C_{t}\right)-\left(1-D_{t}-\kappa I_{t}\right) \frac{L_{t}^{1+\eta}}{1+\eta}-u_{\kappa} \kappa I_{t}-u_{d} D_{t}
$$

and

$$
\left(1-D_{t}\right) C_{t}=\left(1-D_{t}-\kappa I_{t}\right)\left(L_{t}-\frac{\chi\left(\bar{M}_{t}\right)}{2}\left(M_{t}\right)^{2}\right)
$$

The Lagrangian is

$$
\begin{aligned}
V_{t}\left(I_{t}, S_{t}, D_{t}, \bar{M}_{t}\right)= & u_{t}+\beta V_{t+1}+\lambda_{t}\left(\left(1-D_{t}-\kappa I_{t}\right)\left(L_{t}-\chi\left(\bar{M}_{t}\right)\left(M_{t}\right)^{2}\right)-\left(1-D_{t}\right) C_{t}\right) \\
& +\lambda_{e, t}\left(e_{t}-\bar{e}-\left(1-D_{t}\right) e^{c} C_{t}^{2}-\left(1-D_{t}-\kappa I_{t}\right) e^{l}\left(1-M_{t}\right)^{2} L_{t}^{2}\right) \\
& +\lambda_{i, t}\left(I_{t+1}-\gamma e_{t} I_{t} S_{t}-(1-\rho) I_{t}+\delta\left(\kappa I_{t}\right) \kappa I_{t}\right) \\
& +\lambda_{s, t}\left(S_{t+1}-S_{t}+\gamma e_{t} I_{t} S_{t}\right) \\
& +\lambda_{d, t}\left(D_{t+1}-D_{t}-\delta\left(\kappa I_{t}\right) \kappa I_{t}\right)
\end{aligned}
$$


The first order conditions for consumption and labor are then (highlighted in red the difference with the decentralized equilibrium)

$$
\begin{aligned}
& C_{t}: \quad C_{t}^{-1}=\lambda_{t}+2 \lambda_{e, t} e^{c} C_{t} \\
& L_{t}: \quad L_{t}^{\eta}=\lambda_{t}-2 \lambda_{e, t} e^{l}\left(1-M_{t}\right)^{2} L_{t} \\
& M_{t}: \quad \lambda_{t} \chi_{t} M_{t}=\frac{\beta V_{\bar{M}, t+1}}{1-D_{t}-\kappa I_{t}}+2 \lambda_{e, t} e^{l}\left(1-M_{t}\right) L_{t}^{2}
\end{aligned}
$$

The marginal utilities of the planner with respect to exposure are twice as high as those of the private sector because of the contagion externalities: private agents only care about how their behavior affect their own infection risk. They do not care about how their behavior affects the infection risk of others.

The remaining first order conditions are the same as those of the private sector

$$
\begin{aligned}
e_{t} & : \lambda_{e, t}=\left(\lambda_{i, t}-\lambda_{s, t}\right) \gamma I_{t} s_{t} \\
I_{t+1}: \lambda_{i, t} & =-\beta V_{I, t+1} \\
S_{t+1}: \lambda_{s, t} & =-\beta V_{S, t+1} \\
D_{t+1}: \lambda_{d, t} & =-\beta V_{D, t+1}
\end{aligned}
$$

The envelope conditions are

$$
\begin{aligned}
V_{I, t}= & \kappa \frac{L_{t}^{1+\eta}}{1+\eta}-\kappa u_{\kappa}-\kappa \lambda_{t}\left(L_{t}-\frac{\chi_{t}}{2} M_{t}^{2}\right)+\lambda_{e, t} \kappa e^{l}\left(1-M_{t}\right)^{2} L_{t}^{2}-(1-\rho) \lambda_{i, t} \\
& \quad-\gamma e_{t} S_{t} \lambda_{i, t}-\left(\delta_{t} \kappa+\delta_{t}^{\prime} \kappa^{2} I_{t}\right)\left(\lambda_{d, t}-\lambda_{i, t}\right)
\end{aligned}
$$




\section{B Properties of Contagion Dynamics}

\section{B.1 Definitions}

We start with the most basic concept in epidemiology, the basic reproduction number, which we denote by $\mathcal{R}$ because the usual notation "R_not" is terribly confusing. $\mathcal{R}$ is the expected number of cases directly generated by one case when everyone else is susceptible. The most basic model is to assume that when someone is infected there are three stages

1. a latency period $T_{1}$ when the individual is not yet infectious

2. infectious period $T_{2}-T_{1}$

3. recovered period after $T_{2}$ when the individual is not infectious anymore

If the contact rate (exposure) is $e$ and the probability of infection conditional on contact is $\gamma$, the expected number of secondary cases per primary case in a fully susceptible population is therefore

$$
\mathcal{R}=\gamma e\left(T_{2}-T_{1}\right)
$$

In our notations, e is the number of people that one individual meets per unit of time and $\gamma$ is the probability of transmitting the disease conditional on a meeting between one infectious and one susceptible agent.

\section{B.2 SIR Model}

The $S I R$ model builds on this idea. We define the length of one period so that $T_{1}=1$. If someone is infected in period $t$, then she will start spreading the disease in period $t+1$. Let $I_{t}$ be the number of infected individuals and $S_{t}$ the number of susceptible individuals at the beginning of time $t$ in a population of size $N$. Each infected agent meets $e$ people. We assume that the meetings are random and that the population is always evenly mixed, therefore the probability of meeting a susceptible person is $S / N$. The number of meetings between infected and susceptible agents is therefore $e I S / N$ and the total number of new infections is $\gamma e I_{t} \frac{S_{t}}{N}$. In our macro model $e$ is an endogenous variable but we take it as a constant for now. We assume that recovery follows a Poisson process with intensity $\rho$. The 
infection equation is then

$$
I_{t+1}=\gamma e I_{t} \frac{S_{t}}{N}+(1-\rho) I_{t}
$$

Consider a population of size $N$ (large) of initially susceptible individuals $\left(S_{0}=N\right)$. If one individual is infected, the total number of secondary infections from that individual is

$$
\mathcal{R}=\sum_{\tau=0}^{\infty} \gamma e(1-\rho)^{\tau}=\frac{\gamma e}{\rho}
$$

Note that $\mathcal{R}$ is a number, not a rate per unit of time. The model has a steady state at $I=0$ and $S=1$ but it is unstable in the sense that if one individual gets infected the system converges to a different steady state. In general we can write

$$
\frac{I_{t+1}}{I_{t}}=1+\gamma e \frac{S_{t}}{N}-\rho
$$

When $S / N \approx 1$, the number infected people evolves exponentially as $\frac{I_{t+1}}{I_{t}} \approx 1+\gamma e-\rho$. If $R_{0}<1$ then a small infection disappears exponentially. If $R_{0}>1$ then there is an epidemic where $I$ initially grows over time. The growth continues as long as $\gamma e \frac{S_{t}}{N}>\rho$. Eventually the number of susceptible people decreases and growth slows down or reverses, depending on how we close the model.

There are two ways to close the model. The simpler one, called the $S I S$ model, assumes that recovered agents $\rho I$ go back to the pool of susceptible agents. This is the model used to study the common cold. In that case $N=S_{t}+I_{t}$ and the equation becomes

$$
I_{t+1}=\gamma e I_{t} \frac{N-I_{t}}{N}+(1-\rho) I_{t}
$$

and the steady state infection rate is $\frac{I}{N}=\max \left(0 ; 1-\frac{\rho}{\gamma e}\right)$.

The other way is to introduce a population of recovered agents $R$ who are not susceptible anymore. This model - called $S I R$ - is used for flu epidemics, among others. In the simple 
model, $R$ is an absorbing state. The system becomes

$$
\begin{aligned}
S_{t+1}-S_{t} & =-\gamma e I_{t} \frac{S_{t}}{N} \\
R_{t+1}-R_{t} & =\rho I_{t}
\end{aligned}
$$

and of course $N=S_{t}+I_{t}+R_{t}$. Note that $S$ is (weakly) decreasing and $R$ is (weakly) increasing, therefore their limits exist. Since $N=S_{t}+I_{t}+R_{t}$ so does the limit of $I$. For $R$ and $S$ to be constant it must be that $I$ tends to zero. Therefore

$$
\lim _{t \rightarrow \infty} I_{t}=0
$$

That is the first simple property of the solution. Second, since $S$ is decreasing, $I$ must be (at most) single-peaked. If $I_{0}$ is small and $R_{o}>1$ then $I_{t}$ must grow, reach a maximum, and then decrease towards zero. This is the typical shape of the curves found in the literature. Harko et al. (2014) provide an analytical solution to the differential equations (in continuous time).

Let us now study the long run behavior of $S$ and $R$. Combining equations (3) and (4) we get

$$
R_{t+1}-R_{t}=-N \frac{\rho}{\gamma e} \frac{S_{t+1}-S_{t}}{S_{t}}
$$

This equation is simpler to write in continuous time

$$
\frac{\dot{S}}{S}=-\frac{\gamma e}{\rho} \frac{\dot{R}}{N}
$$

and to integrate the solution:

$$
\log \left(\frac{S_{t}}{S_{0}}\right)=-\frac{\gamma e}{\rho}\left(\frac{R_{t}-R_{0}}{N}\right)
$$

This equation holds along any transition path without exogenous shocks. In the limit, since 
$S_{\infty}+R_{\infty}=1$ we have the transcendental equation

$$
\log \left(\frac{S_{\infty}}{S_{0}}\right)=-\frac{\gamma e}{\rho}\left(\frac{1-S_{\infty}-R_{0}}{N}\right)
$$

The long run steady state $\left(S_{\infty}, R_{\infty}\right)$ depends on the initial conditions as well at the basic reproduction number $\mathcal{R}$. We can summarize our discussion in the following Lemma.

Lemma. The SIR model is fully characterized by $\mathcal{R}=\frac{\gamma e}{\rho}$ and the initial conditions $\left(S_{0}, R_{0}\right)$. If $\mathcal{R}<1$, infections die out without epidemic. If $\mathcal{R}>1$, a small infection $I_{0}$ creates an epidemic: It rises, reaches a maximum in finite time before declining towards zero: $I_{\infty}=0$. The long run limits $S_{\infty}$ and $R_{\infty}$ exist and satisfy $S_{\infty}+R_{\infty}=1$ and $\log \left(\frac{S_{\infty}}{S_{0}}\right)=-\mathcal{R}\left(\frac{1-S_{\infty}-R_{0}}{N}\right)$.

The complete model takes into account that some individuals will die from the disease. We assume that a fraction $\kappa$ of infected agents become (severely) sick and a fraction $\delta$ of the sick patients die. Hence we have another absorbing state, $D$. The system of equation of the SIRD model becomes

$$
\begin{aligned}
I_{t+1} & =\gamma I_{t} \frac{S_{t}}{N}+(1-\rho-\delta \kappa) I_{t} \\
S_{t+1} & =S_{t}-\gamma I_{t} \frac{S_{t}}{N} \\
R_{t+1} & =R_{t}+\rho I_{t} \\
D_{t+1} & =D_{t}+\delta \kappa I_{t}
\end{aligned}
$$

The number of sick people is $\kappa I_{t}$ and determines the pressure on the health care system. From the perspective of the epidemic we could aggregate $D$ and $R$ into one absorbing state: $\tilde{R}=D+R$ such that $\tilde{R}_{t+1}=\tilde{R}_{t}+(\rho+\delta \kappa) I_{t}$. The long run solution is

$$
\log \left(\frac{S_{\infty}}{S_{0}}\right)=-\frac{\gamma e}{\rho+\delta \kappa}\left(\frac{1-S_{\infty}-\tilde{R}_{0}}{N}\right)
$$

and $\tilde{R}_{\infty}=1-S_{\infty}$ while $D_{\infty}=D_{0}+\frac{\delta \kappa}{\delta \kappa+\rho}\left(\tilde{R}_{\infty}-\tilde{R}_{0}\right)$. From an economic and social perspective we need to keep track of $D$ and $R$ separately in any case. 


\section{B.3 SIR model with exogenous birth and death}

The path dependence of the long run steady state is a somewhat artificial consequence of the lack of entry and exit. Suppose that $\epsilon N$ people are both in state $S$ each period, and also that there is a constant exogenous death rate $\epsilon$. The system is

$$
\begin{aligned}
I_{t+1} & =\gamma e I_{t} \frac{S_{t}}{N}+(1-\rho-\epsilon) I_{t} \\
S_{t+1} & =(1-\epsilon) S_{t}-\gamma e I_{t} \frac{S_{t}}{N}+\epsilon N \\
R_{t+1} & =(1-\epsilon) R_{t}+\rho I_{t}
\end{aligned}
$$

Note that population is constant: $S_{t+1}+I_{t+1}+R_{t+1}=N$. Now the steady state requires

$$
\begin{aligned}
\gamma e \frac{S}{N} I & =I(\epsilon+\rho) \\
\gamma e I \frac{S}{N} & =\epsilon(N-S) \\
\rho I & =\epsilon R
\end{aligned}
$$

Since $I>0$ we can easily solve for the unique steady state

$$
\begin{aligned}
& \frac{S}{N}=\frac{\epsilon+\rho}{\gamma e} \\
& \frac{I}{N}=\epsilon \frac{1-\frac{\epsilon+\rho}{\gamma e}}{\epsilon+\rho} \\
& \frac{R}{N}=\rho \frac{1-\frac{\epsilon+\rho}{\gamma e}}{\epsilon+\rho}
\end{aligned}
$$

And now we can take the limit as $\epsilon \rightarrow 0$ to get $\frac{I}{N}=0, \frac{S}{N}=\frac{\rho}{\gamma e}=\mathcal{R}^{-1}$ and $\frac{R}{N}=1-\frac{\rho}{\gamma e}=$ $1-\mathcal{R}^{-1}$. Adding a small amount of exogenous birth and death renders the long run steady state independent of initial conditions. 